\title{
Distributed Channel Synthesis
}

\author{
Paul Cuff
}

\begin{abstract}
Two familiar notions of correlation are rediscovered as the extreme operating points for distributed synthesis of a discrete memoryless channel, in which a stochastic channel output is generated based on a compressed description of the channel input. Wyner's common information is the minimum description rate needed. However, when common randomness independent of the input is available, the necessary description rate reduces to Shannon's mutual information. This work characterizes the optimal trade-off between the amount of common randomness used and the required rate of description. We also include a number of related derivations, including the effect of limited local randomness, rate requirements for secrecy, applications to game theory, and new insights into common information duality.

Our proof makes use of a soft covering lemma, known in the literature for its role in quantifying the resolvability of a channel. The direct proof (achievability) constructs a feasible joint distribution over all parts of the system using a soft covering, from which the behavior of the encoder and decoder is inferred, with no explicit reference to joint typicality or binning. Of auxiliary interest, this work also generalizes and strengthens this soft covering tool.
\end{abstract}

Index Terms-Channel simulation, channel synthesis, soft covering, common information, random number generator, resolvability, reverse Shannon theorem, total variation distance.

\section{INTRODUCTION}

$\mathbf{W}$ HAT is the intrinsic connection between correlated random variables? How much interaction is necessary to create correlation? These are some of the inquiries that are illuminated by the distributed channel synthesis problem, introduced as follows: An observer (encoder) of a random i.i.d. source sequence $X_{1}, X_{2}, \ldots$ describes the sequence to a distant random number generator (decoder) that produces $Y_{1}, Y_{2}, \ldots$. What is the minimum rate of description needed to achieve a joint distribution that is statistically indistinguishable (as measured by total variation) from the distribution induced by a memoryless channel?

The nature of distributed channel synthesis is quite different than most problems in communication and source coding. The objective of mimicking a random process is significantly more stringent than, say, producing an output sequence that is empirically correlated (jointly typical) with the input sequence. Here we require the resulting input-output pairs $\left(X_{t}, Y_{t}\right)$ to be nearly i.i.d. according to the joint distribution that a prescribed memoryless channel would imply. In previous work [2] we define two notions of coordination that distinguish this important point. This work is the "strong coordination" of [2].

Remarkably, random bits available in common to both the encoder and decoder play a non-trivial role in channel

This work is supported by the National Science Foundation (grant CCF1116013) and the Air Force Office of Scientific Research (grant FA9550-12$1-0196)$.

P. Cuff (cuff@princeton.edu) is with Department of Electrical Engineering at Princeton University.

This paper was presented in part at ISIT 2008 [1]. synthesis. Because of the unusual nature of the problem, common randomness can replace some (yet not all) of the communication, providing a stochastic connection between the encoder and the decoder. This stems from an important property of channel synthesis-unpredictability. A properly synthesized channel will produce random outputs, free from perceivable patterns to all who do not see the communication and common randomness.

A particularly enticing use of distributed channel synthesis is in interactive adversarial settings. In the context of game theory, correlated strategies can be advantageous to cooperating participants. Correlation constraints on actions have been considered in the literature (e.g. [3] and [4]). We discuss the role of our channel synthesis results and the connection to secrecy systems in §I-B In many repeated game settings, distributed channel synthesis provides the optimal means of communication.

The distributed channel synthesis problem provides a fresh look at correlation. Many fruitful efforts have been made to quantify correlation between two random variables. Each quantity is justified by the operational questions that it answers. Covariance dictates the mean squared-error in linear estimation. Shannon's mutual information is the descriptive savings for lossless compression due to side information and the additional growth rate of wealth in investing. Gács and Körner's common information [5] is the number of common random bits that can be extracted from correlated random variables. It is less than mutual information. Wyner's common information [6] is the number of common random bits needed to generate correlated random variables and is greater than mutual information.

In the distributed channel synthesis problem, two quantities emerge as extreme points - Shannon's mutual information and Wyner's common information. Without common randomness, the required communication rate is Wyner's common information $C(X ; Y)$, consistent with Wyner's result in [6] concerning the minimum connection needed to generate correlated random variables. However, when enough common randomness is available, the communication requirement is reduced to the mutual information $I(X ; Y)$ (consistent with [7]). These extremes are evident in the main result, Theorem II.1 and common information is discussed further in

Channel synthesis has emerged recently as a concept of interest in quantum and classical information theory. Soljanin [8] studied this in the context of quantum compression with unlimited common randomness. Bennett et. al. introduced a "reverse Shannon theorem" [7] (see also [9], [10], and [11]) which states that all channels of the same capacity are equally valuable. If one ignores encoding complexity and has unlimited common randomness available, then any memoryless channel can be used to synthesize any other channel of lower 
capacity. Referring to Shannon's theorem as the reduction of a noisy channel to a noise-free one, their reverse Shannon theorem uses a noise-free channel to synthesize a noisy one. This is precisely the problem considered in the present paper as well; however, we consider common randomness also to be a limited resource, yielding a trade-off between the use of communication and common randomness.

Limited common randomness for distributed channel synthesis was considered by Winter in [12] for a certain extremal operating point rather than the entire optimal trade-off. Winter's communication scheme does not immediately generalize, though some of the proof methods were similar to ours. He then connected these quantities (communication and common randomness for channel synthesis) to so-called "extrinsic" and "intrinsic" data in quantum measurements [13]. Further work on quantum measurements and exact channel synthesis can be found in [14], [15], and [16].

In addition to the main result, an emphasis of this paper is the proof technique. Our construction of optimal codecs in is unusual in that we don't begin by stating the behavior of the encoder in an explicit, causal manner but instead construct a joint distribution and infer the encoder behavior from it. The tool that we use is the soft covering lemma of $₫ \mathrm{IV}$ This is essentially the same tool used for the achievability proofs of Wyner's common information [6], the resolvability of a channel [17], and other results in the literature. In \$VII] we develop and generalize this tool, showing for example how it can be extended to superposition codebooks, similar to [18]. Recently an alternative proof tool has been proposed in [19] which uses a random binning construction to take the role of soft covering.

We provide the main result and examples in $₫$ followed by a number of extensions to the basic distributed channel synthesis problem in Other extensions to this problem can be found in the recent literature (e.g. [18], [20], [21], [22]), building on our introduction of the problem in [1], [23], and [2].

Let us note that ultimately the main result of the present paper has been solved concurrently and independently by Bennett, Devetak, Harrow, Shor, and Winter and can be found in preprint form in [10]. A presentation given by Bennett [24] of unpublished work, unknown to us at the time, contains the complete trade-off between communication and common randomness and occurred at roughly the same time as our publication in [1].

\section{MAIN RESULT}

\section{A. Distributed Channel Synthesis}

Let $\left\{X_{i}\right\}$ be a discrete i.i.d. random process, distributed according to $Q_{X}$. The dashed box in Fig. 1 represents a system that is designed to operate as if it were a memoryless channel defined by the conditional probability mass function $Q_{Y \mid X}$. However, the internal components of the box are constrained. Suppose the input and output of the channel are not co-located. An encoder which observes the channel input and a decoder which produces the channel output use communication and common randomness to synthesize the channel. The synthesis is successful if it cannot be distinguished through a statistical test from the memoryless channel that it is designed to mimic. This requirement will be clarified in

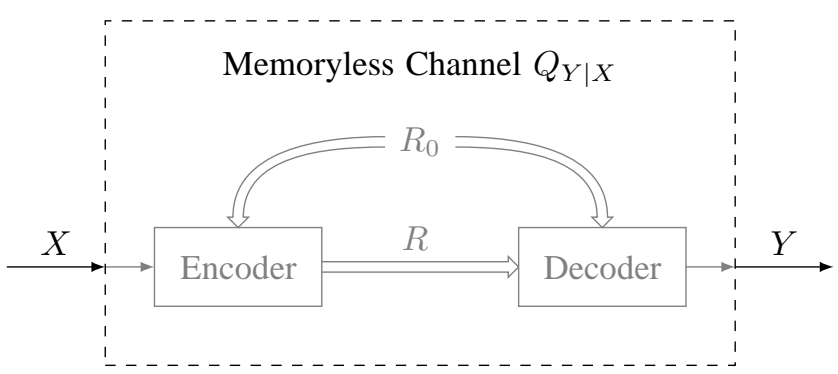

Fig. 1: Main Setup: We synthesize a memoryless channel across a distance by making use of communication and common randomness. The necessary and sufficient rates are given in Theorem $\Pi$ I.1

The distributed channel synthesis problem asks what resources are needed to successfully accomplish this channel synthesis. The resources come in the form of a message $J$ that is transmitted from the encoder to the decoder and common randomness $K$ that is independent of the channel input. This work characterizes the required bit-rates of this communication and common randomness.

We allow the system to operate on blocks of $n$ inputs at a time, producing $n$ channel outputs. This is the standard block encoding used in communication and compression. However, even within each block, the system mimics a memoryless channel, where the $n$ outputs are conditionally independent given the $n$ inputs.

Definition 1. The desired input-output distribution for a block-length $n$ is the product distribution on the pair of sequences $X^{n}$ and $Y^{n}$ specified by the probability mass function $\prod_{t=1}^{n} Q_{X}\left(x_{t}\right) Q_{Y \mid X}\left(y_{i} \mid x_{i}\right)$. We abbreviate this simply as

$$
\prod Q_{X} Q_{Y \mid X}
$$

\section{B. Encoder and Decoder}

For a block-length $n$, the encoder produces a description of the source sequence $X^{n}$ at rate $R$, represented by $J \in\left[2^{n R}\right] \triangleq$ $\left\{1, \ldots, 2^{n R}\right\}$. A random variable $K$, uniformly distributed on $\left[2^{n R_{0}}\right]$ and independent of $X^{n}$, represents the common random bits at rate $R_{0}$ known at both the encoder and decoder. The decoder generates a channel output $Y^{n}$ based only on $J$ and $K$.

The encoder and decoder are free to use randomization, and indeed they benefit from doing so. Accordingly, the encoder and decoder are described by conditional probability mass functions.

Encoder: $\quad F_{J \mid X^{n}, K}$ (a probability distribution),

Decoder: $\quad G_{Y^{n} \mid J, K}$ (a probability distribution).

Definition 2. An $\left(R, R_{0}, n\right)$ channel synthesis code for input alphabet $\mathcal{X}$ and output alphabet $\mathcal{Y}$ consists of an encoder $F_{J \mid X^{n}, K}$ and a decoder $G_{Y^{n} \mid J, K}$ defined on the supports $X^{n} \in \mathcal{X}^{n}, Y^{n} \in \mathcal{Y}^{n}, J \in\left[2^{n R}\right]$, and $K \in\left[2^{n R_{0}}\right]$. 


\section{Induced Distribution}

Aside from the common randomness $K$, the behavior of the encoder and the decoder are independent. Therefore, the combined behavior of the encoder and decoder results in a conditional distribution of the message $J$ and output $Y^{n}$ given by

$$
P_{Y^{n}, J \mid X^{n}, K}=F_{J \mid X^{n}, K} G_{Y^{n} \mid J, K} .
$$

Definition 3. The induced joint distribution of an $\left(R, R_{0}, n\right)$ channel synthesis code is the joint distribution on the quadruple $\left(X^{n}, Y^{n}, J, K\right)$ resulting from applying the encoder and decoder to the channel input and common randomness. In other words, it is the probability mass function

$$
P_{X^{n}, Y^{n}, J, K}=P_{Y^{n}, J \mid X^{n}, K} P_{X^{n}, K},
$$

where, by definition of the problem,

$$
P_{X^{n}, K}\left(x^{n}, k\right)=\frac{1}{2^{n R_{0}}} \prod_{t=1}^{n} Q_{X}\left(x_{t}\right) .
$$

Definition 4. The induced input-output distribution is the marginal distribution of the induced joint distribution, assigning joint probabilities to only the input $X^{n}$ and the output $Y^{n}$ as

$$
P_{X^{n}, Y^{n}}\left(x^{n}, y^{n}\right)=\sum_{j, k} P_{X^{n}, Y^{n}, J, K}\left(x^{n}, y^{n}, j, k\right) .
$$

\section{Tolerance}

We say that the memoryless channel specified by $Q_{Y \mid X}$ can be synthesized with rates $\left(R, R_{0}\right)$ for input distribution $Q_{X}$ if there exists an $\left(R, R_{0}, n\right)$ channel synthesis code that induces the desired input-output distribution $\prod Q_{X} Q_{Y \mid X}$. However, we actually tolerate some error. If we require exact synthesis then we forfeit some of the substantial benefit that compression provides. For example, consider distributed synthesis of the identity channel, which is equivalent to lossless compression. "Near lossless" compression of $\left\{X_{i}\right\}$ can be achieved with a rate of $R=H(X)$, but exact lossless compression (and exact synthesis of the identity channel) requires $R=\log |\mathcal{X}|$.

Rather than tolerate error in the channel synthesis, we might instead ask for exact synthesis using variable length communication, just as variable length codes such as Huffman codes allow for exact lossless compression while achieving efficient average description lengths. For distributed channel synthesis, a simple adaptation to block encoding would be to use an efficient channel synthesis code most of the time, which nearly synthesizes the channel, and with a small probability use an inefficient channel synthesis code (uncompressed communication) to implement the needed correction. This is similar to the approach taken in [7], where exact synthesis is achieved using variable rate communication and an unlimited supply of common randomness. Also, exact synthesis is achieved in [15] using rejection sampling. But the steps for achieving efficient exact synthesis in our case of limited common randomness are not immediately obvious.

Instead of exact synthesis, we tolerate an arbitrarily small error measured by total variation. In $₫$ II-I we define and discuss total variation as a meaningful metric of tolerance. For now, we move directly to the main definition for this work.

Definition 5. A pair of rates $\left(R, R_{0}\right)$ is achievable for synthesizing a memoryless channel specified by $Q_{Y \mid X}$ with input distribution $Q_{X}$ if there exists a sequence of $\left(R, R_{0}, n\right)$ channel synthesis codes, for $n=1,2, \ldots$, where

$$
\lim _{n \rightarrow \infty}\left\|P_{X^{n}, Y^{n}}-\prod Q_{X} Q_{Y \mid X}\right\|_{T V}=0 .
$$

Let $\mathcal{C}$ be the closure of the set of achievable rate pairs $\left(R, R_{0}\right) \cdot 1$

$$
\mathcal{C} \triangleq \text { Closure }\left\{\text { Achievable }\left(R, R_{0}\right) \text { for } Q_{X}, Q_{Y \mid X}\right\} .
$$

\section{E. Main Result}

The main result of this paper characterizes the rate of communication and rate of common randomness needed to synthesize a discrete memoryless channel $Q_{Y \mid X}$ with an i.i.d. input distribution $Q_{X}$. This characterization is given in the definition of the following set $\mathcal{S}$ :

$$
\mathcal{S} \triangleq\left\{\begin{aligned}
\left(R, R_{0}\right) \in \mathcal{R}^{2} & : \exists P_{X, Y, U} \in \mathcal{D} \text { s.t. } \\
R & \geq I(X ; U), \\
R_{0}+R & \geq I(X, Y ; U) .
\end{aligned}\right\},
$$

where

$$
\mathcal{D} \triangleq\left\{\begin{array}{ll}
P_{X, Y, U}: & (X, Y) \sim Q_{X} Q_{Y \mid X} \\
& X-U-Y \text { Markov } \\
& |\mathcal{U}| \leq|\mathcal{X}||\mathcal{Y}|+1 .
\end{array}\right\} .
$$

Theorem II.1. For a discrete memoryless channel,

$$
\mathcal{C}=\mathcal{S}
$$

Furthermore, the total variation of (6) decays exponentially fast with $n$ in the interior of $\mathcal{C}$.

Two extreme points of the rate region $\mathcal{S}$ for distributed channel synthesis are manifested directly in the inequalities of (8) and illustrated in Fig. 2. If $R_{0}=0$, the second inequality dominates, and the minimum communication rate $R$ is Wyner's common information [6], defined as

$$
C(X ; Y) \triangleq \min _{U: X-U-Y} I(X, Y ; U) .
$$

At the other extreme, we see that with unlimited common randomness, the communication requirement reduces to $R \geq I(X ; Y)$. To see this, notice that the data processing inequality, which yields $I(X ; U) \geq I(X ; Y)$, can be met with equality by selecting $U=Y$. Furthermore, this implies that $I(X, Y ; Y)-R=H(Y)-I(X ; Y)=H(Y \mid X)$ is a sufficient rate of common randomness to minimize the communication rate requirement. It turns out, as is shown in [2], that sometimes a different choice of $U$ also minimizes the communication requirement while requiring even less common randomness. The smallest amount of common randomness (after which additional common randomness does not benefit) is referred to as necessary conditional entropy in [2]:

$$
H(Y \dagger X) \triangleq \min _{f: X-f(Y)-Y} H(f(Y) \mid X) .
$$

\footnotetext{
${ }^{1}$ We deal with the closure because our proof does not handle the boundary points.
} 


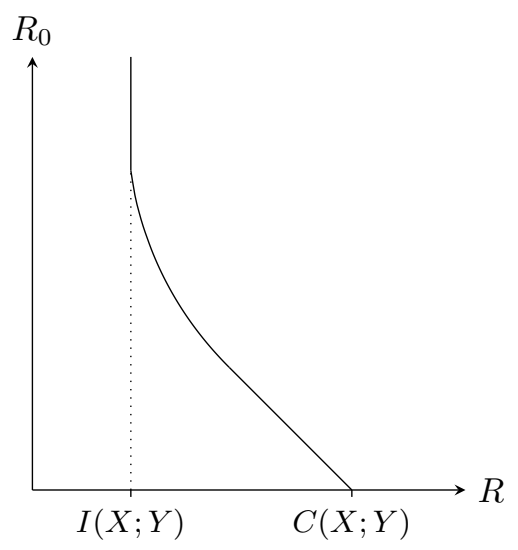

Fig. 2: Main Result: Theorem II.1 gives a trade-off between the rate of communication and the rate of common randomness required to synthesize a discrete memoryless channel. At the extremes, with no common randomness the communication rate requirement is Wyner's common information $C(X ; Y)$, and the requirement reduces to the mutual information $I(X ; Y)$ when unlimited common randomness is available.

Proof of Theorem [I.1 is the subject of sections IV] V] and VI] The achievability proof holds for general memoryless sources and channels, not only those that are discrete. However, the converse presented in this work is specific to finite alphabets. A general converse might arise from a careful analysis of $\mathcal{S}$. For example, it would be sufficient to show that $\cap_{[Q]} \mathcal{S}_{[Q]} \subset \mathcal{S}$, where $[Q]$ represents the distribution of a finite quantization of $X$ and $Y$ under the desired distribution $Q_{X, Y}$, and $\mathcal{S}_{[Q]}$ is defined as $\mathcal{S}$ in (8) and (9) but with respect to $[Q]$. By Theorem $\llbracket .1$ and first principles, $\mathcal{C} \subset \mathcal{S}_{[Q]}$ for any $[Q]$.

\section{F. Example: Erasure Channel}

Let $Q_{X}$ be the binary symmetric distribution (i.e. Bernoullihalf), and consider the symmetric erasure channel $Q_{Y \mid X}$ with erasure probability $p$. We now find the optimal distributed channel synthesis rates $\left(R, R_{0}\right) \in \mathcal{S}$ by considering Markov distributions in $\mathcal{D}$. Fortunately, the sparsity in the joint distribution $Q_{X, Y}$ simplifies this optimization.

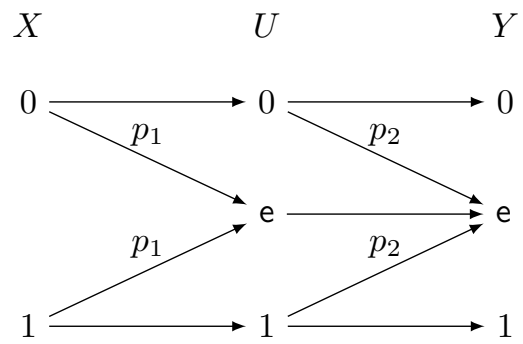

Fig. 3: Concatenated Erasure Channels: Any optimal point in the rate region $\mathcal{S}$ in (8) for the symmetric erasure channel is achieved with a choice of $U$ that constitutes a concatenation of two symmetric erasure channels.

In the appendix we show that the optimizing choices of $P_{X, Y, U}$ will be the concatenation of two erasure channels depicted in Fig. 3. We are left with two parameters-the erasure probability $p_{1}$ of the first channel $P_{U \mid X}$ and the erasure probability $p_{2}$ of the second channel $P_{Y \mid U}$-and one constraint: $\left(1-p_{1}\right)\left(1-p_{2}\right)=(1-p)$. By labeling $r=\left(1-p_{1}\right)$ and evaluating the mutual information terms, we obtain the following achievable rate region:

$$
\mathcal{S}=\left\{\begin{aligned}
\left(R, R_{0}\right) & \in \mathcal{R}^{2}: \\
\exists r & \in\left[1-p, r^{*}\right] \text { such that } \\
R & \geq r \text { bits, } \\
R_{0}+R \geq & h(p) \\
& +r\left(1-h\left(\frac{1-p}{r}\right)\right) \text { bits. }
\end{aligned}\right\}
$$

where $r^{*}=\min \{2(1-p), 1\}$ and $h(\cdot)$ is the binary entropy function. Choices of $r>r^{*}$ are suboptimal.

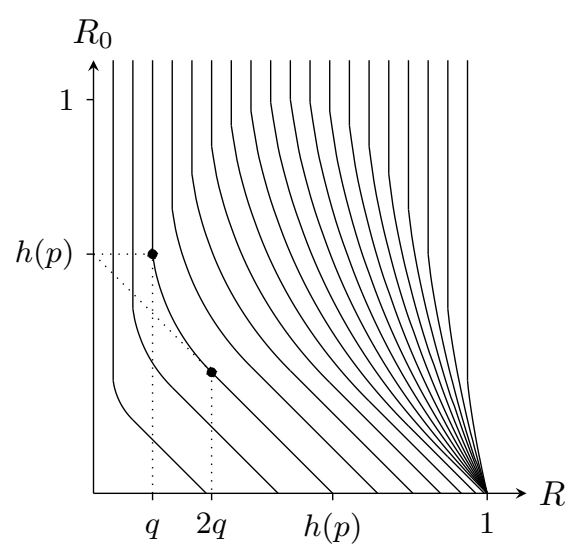

Fig. 4: Erasure Channel Rate Regions: The boundaries of the achievable rate regions for synthesis of the $p$-erasure channel with symmetric inputs are shown for $p=.05, .1, .15, \ldots, .9, .95$ from right to left. Transition points on the curve for $p=.85$ are labeled, where $q=1-p$.

Common Information: The common information $C_{Q}(X ; Y)$ is found by evaluating the second inequality of (13) (representing $I(X, Y ; U))$ at $r=r^{*}$. For erasure probabilities $p \leq 1 / 2$ we see that $r^{*}=1$, which is equivalent to choosing $U=X$. The common information in this case is $C_{Q}(X ; Y)=1$ bit. For erasure probabilities $p>1 / 2$ we get $r^{*}=2(1-p)$, which is equivalent to choosing the channel $P_{Y \mid U}$ to have $50 \%$ erasures. The common information in this case is $C_{Q}(X ; Y)=h(p)$. Notice that it is easily verified in Fig. 3 that $H(X, Y \mid U=u) \leq 1$ bit for each $u$, which is achieved with equality by $r^{*}$. To summarize:

$$
C_{Q}(X ; Y)= \begin{cases}1 \text { bit, } & p<1 / 2, \\ h(p), & p \geq 1 / 2 .\end{cases}
$$

Minimum Communication: The minimum communication rate required in the presence of enough common randomness is $R \geq I_{Q}(X ; Y)=1-p$ bits, and the rate of common randomness needed to achieve this is $R_{0} \geq H_{Q}(Y \dagger X)=$ $H_{Q}(Y \mid X)=h(p)$. This operating point corresponds to a simple synthesis strategy. The common randomness can be used to generate a list of erasure locations, and the encoder can then transmit the non-erased bits. 


\section{G. Example: Reverse Erasure Channel}

Now consider the reverse of the erasure channel example, by switching the input and output, as depicted in Fig. 5. The channel input distribution $Q_{X}$ is symmetric on the set $\{0, e, 1\}$ with probability $p$ of erasure. The channel sorts the erasures randomly into 0's and 1's.

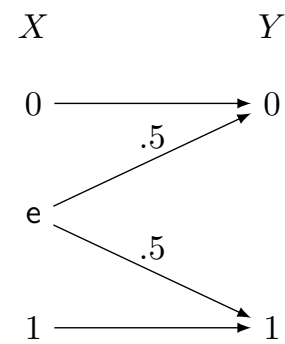

Fig. 5: Reverse Erasure Channel.

The same derivation and parameterizations as above (erasure channel) hold for this example as well. The only modifications to $\mathcal{S}$ are the range of the optimal values of $r$ and the necessary update to the first inequality:

$$
\mathcal{S}=\left\{\begin{array}{rll}
\left(R, R_{0}\right) & \in \mathcal{R}^{2}: \\
\exists r & \in\left[r^{*}, 1\right] \text { such that } \\
R \geq & h(p)-r h\left(\frac{1-p}{r}\right) \\
& +(1-p) \text { bits, } \\
R_{0}+R \geq & h(p)-r h\left(\frac{1-p}{r}\right) \\
& +r \text { bits. }
\end{array}\right\},
$$

where $r^{*}=\min \{2(1-p), 1\}$ and $h(\cdot)$ is the binary entropy function. Choices of $r<r^{*}$ are suboptimal.

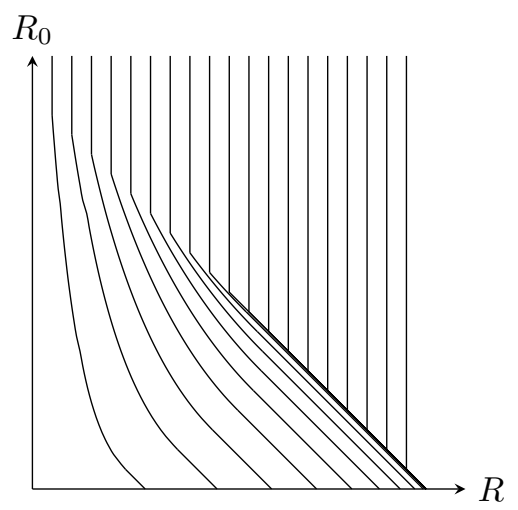

Fig. 6: Reverse Erasure Channel Rate Region: The boundaries of the achievable rate regions for synthesis of the reverse $p$ erasure channel with symmetric inputs are shown for $p=$ $.05, .1, .15, \ldots, .9, .95$ from right to left.

\section{H. Example: Scatter Channel}

Consider a channel $Q_{Y \mid X}$ which acts on an input $X \in$ $\{1,2, \ldots, m\}$ and produces an output uniformly at random from the same set excluding $X$. That is,

$$
Q_{Y \mid X}(y \mid x)=\frac{1}{m-1} \mathbf{1}(x \neq y)
$$

where $\mathbf{1}$ is the indicator function. Now, apply to this channel the uniform input distribution $Q_{X}$, and the result is a desired input-output distribution $Q_{X} Q_{Y \mid X}$ that is uniform over all pairs $(X, Y)$ such that $X \neq Y$. This distribution is studied as an example in [25], [26], and [2].

We find the optimal rates $\left(R, R_{0}\right) \in \mathcal{S}$ by considering Markov distributions in $\mathcal{D}$. As in the previous examples, the sparsity in the joint distribution $Q_{X, Y}$ simplifies this optimization.

For any $P_{X, Y, U} \in \mathcal{D}$, the Markov property constrains that for each value $u$ in the support of $U$, the conditional distribution $P_{X, Y \mid U=u}$ is a product distribution $P_{X \mid U=u} P_{Y \mid U=u}$. We categorize these into $(m-1)$ categories based on the support size of $P_{X \mid U=u}$. Call this support $\mathcal{X}_{u}$ with size $a_{u}=\left|\mathcal{X}_{u}\right|$. Then $\mathcal{X}_{u} \cap \mathcal{Y}_{u}=\emptyset$ to avoid any probability that $X=Y$, resulting in $\left|\mathcal{Y}_{u}\right| \leq m-a_{u}$.

For each of the above categories, associated with $a_{u}=$ $1, \ldots, m-1$, we have a trivial bound on conditional entropy:

$$
\begin{aligned}
H(X \mid U=u) & \leq \log a_{u}, \\
H(X, Y \mid U=u) & \leq \log a_{u}+\log \left(m-a_{u}\right) .
\end{aligned}
$$

Thus, $(H(X \mid U), H(X, Y \mid U))$ must be in the convex hull of the union of two dimensional regions defined by (17) and (18) for each $a_{u}$. On the other hand, the corner points of these regions can be achieved due to the symmetry of $Q_{X, Y}$. This is accomplished by constructing $m$-choose- $a_{u}$ conditional distributions $P_{X \mid U=u_{i}} P_{Y \mid U=u_{i}}$, one for each support structure consistent with $a_{u_{i}}=a_{u}$. Let the conditional distributions be uniformly distributed over their supports. Also, let $P_{U}$ be uniformly distributed over this set of $u_{i}$. Therefore,

$$
\mathcal{S}=\operatorname{Conv}\left(\left\{\begin{aligned}
\left(R, R_{0}\right) & \in \mathcal{R}^{2}: \\
\exists a & \in[m-1] \text { s.t. } \\
a & \geq m / 2, \\
R & \geq \log \left(\frac{m}{a}\right), \\
R_{0}+R & \geq \log \left(\frac{m(m-1)}{a(m-a)}\right),
\end{aligned}\right\}\right)
$$

where $\operatorname{Conv}(\cdot)$ indicated the convex hull. This region is depicted in Fig. 7 for $m=3,5$, and 7 .

Common Information: The common information for this distribution was calculated in [2] and can be obtained from the above region. Let $\lceil m\rceil_{2}$ represent the value of $m$ rounded up to the nearest even number.

$$
C_{Q}(X ; Y)=2 \text { bits }-\log \left(\frac{\lceil m\rceil_{2}}{\lceil m\rceil_{2}-1}\right) .
$$

Notice that this increases to 2 bits as the alphabet size $m$ increases.

Minimum Communication: In contract to the common information, the mutual information $I_{Q}(X ; Y)$ decreases to zero as $m$ increases.

$$
\begin{aligned}
I_{Q}(X ; Y) & =\log \left(\frac{m}{m-1}\right) \\
& \approx \frac{1}{m} \log e .
\end{aligned}
$$

The rate of common randomness needed to achieve this minimal communication rate is $R_{0} \geq H_{Q}(Y \dagger X)=H_{Q}(Y \mid X)=$ $\log (m-1)$. 


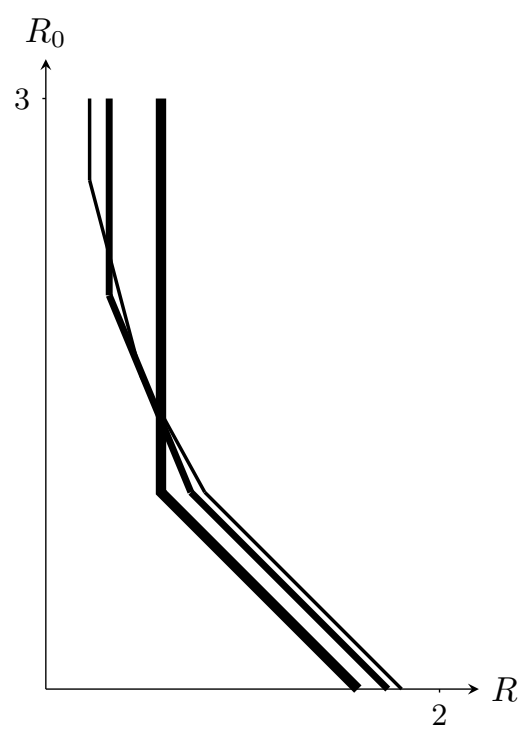

Fig. 7: Scatter Channel Rate Region: The boundaries of the achievable rate regions for synthesis of the scatter channel with symmetric inputs are shown for $|\mathcal{X}|=3,5$, and 7 from thickest to thinnest. Larger alphabets have greater benefit from common randomness.

\section{Total Variation Distance}

We use total variation to measure the distance between the induced input-output distribution and the desired input-output distribution. Total variation between two distributions $\Pi$ and $\Gamma$ on a set $\mathcal{W}$ is defined in the following way:

$$
\begin{aligned}
\|\Pi-\Gamma\|_{T V} & \triangleq \max _{S \subset \mathcal{W}}(\Pi(S)-\Gamma(S)) \\
& =\max _{S \subset \mathcal{W}}(\Gamma(S)-\Pi(S)) .
\end{aligned}
$$

If $\mathcal{W}$ is countable and $\pi(w)$ and $\gamma(w)$ represent the probability mass functions associated with $\Pi$ and $\Gamma$, then

$$
\begin{aligned}
\|\pi-\gamma\|_{T V} & \triangleq \frac{1}{2}\|\pi-\gamma\|_{1} \\
& =\frac{1}{2} \sum_{w \in \mathcal{W}}|\pi(w)-\gamma(w)| \\
& =\|\Pi-\Gamma\|_{T V} .
\end{aligned}
$$

Total variation has properties that make it an attractive measure of tolerance. First is statistical indistinguishability. Consider a test that tries to detect a synthesized channel. The performance of any binary hypothesis test is characterized by two parameters: the probability of false positive $(\alpha)$; and the probability of false negative $(\beta)$. Let $\Gamma$ be the null hypothesis (the channel is genuine) and $\Pi$ be the alternative hypothesis (the channel is synthetic). If the two hypotheses yield identical distributions, then accurate detection is impossible, and $\alpha+\beta=1$ (any value of $\beta=1-\alpha$ can be attained by adjusting the sensitivity of the test). In general,

$$
\alpha+\beta \geq 1-\|\Pi-\Gamma\|_{T V} .
$$

Therefore, if total variation is small, then reliable detection is not possible. This is the objective of channel synthesis.
Another property of total variation is a bound related to expected values of bounded functions.

$$
\left|\mathbf{E}_{\Pi} f(W)-\mathbf{E}_{\Gamma} f(W)\right| \leq 2 f_{\max }\|\Pi-\Gamma\|_{T V},
$$

where $f_{\text {max }}=\max _{w \in \mathcal{W}}|f(w)|$. This bound implies continuity of $\mathbf{E} f(W)$, for bounded $f$, with respect to the distribution $P_{W}$, using total variation as the distance metric. If we know that the total variation is small between two distributions $\Pi$ and $\Gamma$ and we care about the expected value with respect to $\Pi$ of a bounded function, then we are free to instead analyze the expected value with respect to $\Gamma$, which is guaranteed to be nearly equivalent. We use this technique when analyzing the payoff achieved in the game theoretic setting of $₫$ II-B and in related secrecy work in [27], [28], and [29].

Other metrics of distance between probability distributions have been explored in related works, such as [6], [30], and [31]. In particular, Kullback-Leibler divergence $d_{K L}(\Pi ; \Gamma)$ makes an interesting choice of fidelity metric because it is an information-theoretic quantity closely related to other important quantities such as mutual information and, more importantly, because it has implications concerning the asymptotics of hypothesis testing, in the regime of highly reliable detection. Wyner uses K-L divergence, normalized by the block-length, as his tolerance metric for generating correlated random variables in [6].

Kullback-Leibler divergence is a stricter metric than total variation in general-Pinsker's inequality reveals that total variation converges to zero as K-L divergence approaches zero. However, normalized K-L divergence forfeits this relationship. Also, a reverse relationship holds for i.i.d. distributions. That is, if $\Pi \ll \Gamma$ (i.e. $\Pi$ is absolutely continuous with respect to $\Gamma$ ), and $\Gamma$ is an i.i.d. discrete distribution of $n$ variables, then

$$
d_{K L}(\Pi ; \Gamma) \in O\left(\left(n+\log \frac{1}{\mathrm{TV}}\right) \mathrm{TV}\right),
$$

as $\mathrm{TV} \triangleq\|\Pi-\Gamma\|_{T V}$ goes to zero and $n$ goes to infinity 2 In particular, this means that an exponential decay of total variation with respect to $n$ produces an exponential decay in $d_{K L}(\Pi ; \Gamma)$ with the same exponent.

It turns out that the rate region given in the main result (Theorem II.1) no longer holds if the total variation tolerance metric is replaced by Kullback-Leibler divergence in the direction used by Wyner in [6]. That is, let $\Pi$ represent the induced distribution and let $\Gamma$ be the desired distribution. Suppose achievability demands that $d_{K L}(\Pi ; \Gamma)$ be made arbitrarily small (with or without normalization). We again call upon the identity channel as a simple counterexample to Theorem II.1. Notice that $R>H(X)$ is sufficient for the theorem, but $R>\log |\mathcal{X}|$ is necessary for exact lossless compression. Therefore, any rate less than $\log |\mathcal{X}|$ will cause $d_{K L}(\Pi ; \Gamma)=\infty$. On the other hand, for any channel with $Q_{Y \mid X}(y \mid x)>0$ for all $x$ and $y$, the divergence $d_{K L}(\Pi ; \Gamma)$ goes to zero exponentially fast with the same exponent as total variation due to (30).

For a careful comparison of inequalities involving total variation, K-L divergence, and normalized K-L divergence, see [31].

\footnotetext{
${ }^{2}$ This statement uses 29] and [32 Theorem 17.3.3].
} 


\section{EXTENSIONS}

\section{A. Broadcast Channel}

The main result of Theorem II.1 can be readily extended to a situation with multiple separate decoders together synthesizing a memoryless broadcast channel $Q_{Y_{1}, \ldots, Y_{m} \mid X}$, each producing one of the channel output sequences after receiving a common transmission from the encoder as well as common randomness among all nodes. This is depicted in Fig. 8 The region of achievable rates for synthesis is given by

$$
\mathcal{S}_{B C}=\left\{\begin{aligned}
\left(R, R_{0}\right) & \in \mathcal{R}^{2}: \\
\exists P & \in \mathcal{D}_{B C} \text { such that } \\
R & \geq I(X ; U), \\
R_{0}+R & \geq I\left(X, Y_{1}, \ldots, Y_{m} ; U\right) .
\end{aligned}\right\}
$$

where

$$
\mathcal{D}_{B C}=\left\{\begin{aligned}
P_{X, Y_{1}, \ldots, Y_{m}, U} & =P_{X, U} \prod_{i=1}^{m} P_{Y_{i} \mid U}: \\
P_{X, Y_{1}, \ldots, Y_{m}} & =Q_{X} Q_{Y_{1}, \ldots, Y_{m} \mid X} \\
|\mathcal{U}| & \leq|\mathcal{X}|\left|\mathcal{Y}_{1}\right| \ldots\left|\mathcal{Y}_{m}\right|+1 .
\end{aligned}\right\}
$$

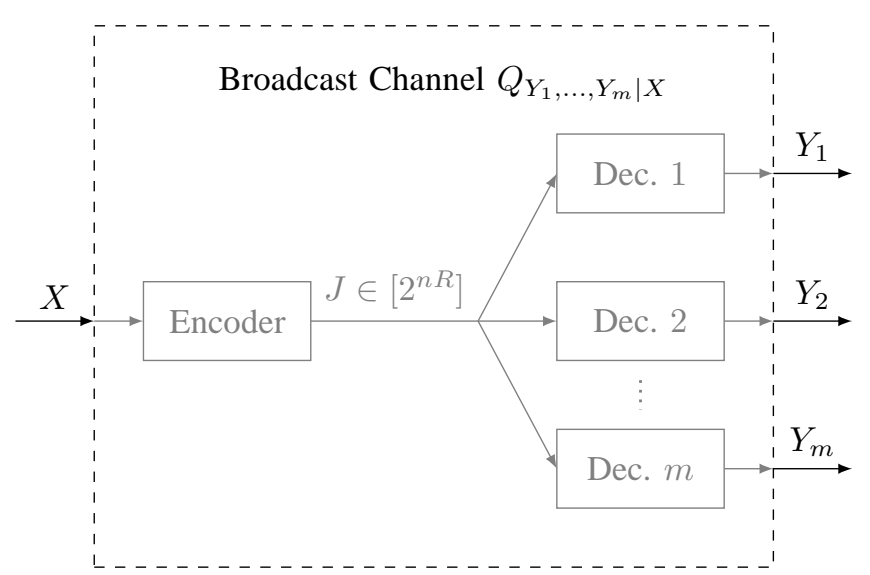

Fig. 8: Broadcast Channel: This setting extends the main result to include the synthesis of a broadcast channel with separated decoders producing each channel output. The decoders each receive a common communication message as well as common randomness.

This region can be proven using the same steps as the main result. Notice that $\mathcal{D}_{B C}$ contains a conditional independence constraint.

\section{B. Game Theory}

Consider a zero-sum repeated game between two teams. Team A consists of two players who on the $t$ th iteration take actions $X_{t} \in \mathcal{X}$ and $Y_{t} \in \mathcal{Y}$. The opponents on Team B take a combined action $Z_{t} \in \mathcal{Z}$. The strategy sets $\mathcal{X}, \mathcal{Y}$, and $\mathcal{Z}$ are finite. The payoff for Team A at each iteration is a timeinvariant finite function $\pi\left(X_{t}, Y_{t}, Z_{t}\right)$ : As a zero-sum game, the payoff for Team B is $-\pi\left(X_{t}, Y_{t}, Z_{t}\right)$. Each participant observes all actions from previous iterations, and each team wishes to maximize its time-averaged expected payoff.

Let Team A play conservatively by assuming the best strategy for Team B. In the worst case (from the viewpoint of Team A), the expected payoff in the $t$ th iteration is

$$
\Pi_{t} \triangleq \min _{z(\cdot, \cdot)} \mathbb{E} \pi\left(X_{t}, Y_{t}, z\left(X^{t-1}, Y^{t-1}\right)\right) .
$$

Clearly (33) could be maximized by finding an optimal mixed strategy $P_{X, Y}^{*}$ that maximizes $\min _{z \in \mathcal{Z}} \mathbb{E}_{P^{*}} \pi(X, Y, z)$ and choosing independent actions accordingly for each iteration. This would correspond to the minimax strategy.

Communication Constraint: Now consider an additional constraint on Team A. The players on Team A have as their only means of coordinating their actions a secure channel of communication, limited to a rate of $R$ bits per game iteration. Specifically, Player 1, who chooses the actions $X_{t}$, communicates at rate $R$ to Player 2, who chooses $Y_{t}$

We say a rate $R$ is achievable for payoff $\Pi$ if there exists a communication protocol that obeys a rate limit of $R$ and produces average expected payoff no less than $\Pi$. That is, there exists a block-length $n$ and a random variable triple $\left(X^{n}, Y^{n}, U\right)$ that has the conditional independence structure $X_{t}-\left(U, X^{t-1}, Y^{t-1}\right)-Y_{t}$ for all $t$ and such that $|\mathcal{U}| \leq 2^{n R}$ and

$$
\frac{1}{n} \sum_{t=1}^{n} \Pi_{t} \geq \Pi
$$

Let $\mathcal{G}$ be the closure of the set of achievable pairs $(R, \Pi)$.

We claim that optimality is obtained by producing i.i.d. actions with respect to a designed joint distribution. Define,

$$
\mathcal{G}_{0} \triangleq\left\{\begin{aligned}
(R, \Pi) & \in \mathcal{R}^{2}: \\
\exists P_{X, Y} & \text { such that } \\
R & \geq C(X ; Y), \\
\Pi & \leq \min _{z \in \mathcal{Z}} \mathbf{E} \pi(X, Y, z) .
\end{aligned}\right\},
$$

where $C(X ; Y)$ is the common information defined in (11).

Lemma III.1 (Optimal cooperative strategy).

$$
\mathcal{G}=\operatorname{Conv}\left(\mathcal{G}_{0}\right) \text {. }
$$

Comments: Notice that Lemma 11 .1 involves a convexification of $\mathcal{G}_{0}$. This means that it may be optimal to split time between two different efficient strategies-one that operates at a low communication rate and one that operates at a high communication rate-in order to satisfy the average rate constraint while competing effectively in the game.

Variants of this problem have been considered in [33], [27], [28], and [29]. The difference in those works is that $X^{n}$ (the actions of Player 1 in this setting) are instead observed states of nature. Their distribution is not designed by Team A. The job of Player 1 is to compress and communicate the observed sequence efficiently to Player 2 . If the communication occurs over a public channel, with use of common randomness to conceal the communication, then the optimal solution is exactly characterized in [28] and is integrally related to the ability to synthesize a memoryless channel. However, communication over a private channel, as in the present setting, is addressed in [33] and still open.

The proof of Lemma $\amalg$.1 is in the appendix.

\footnotetext{
${ }^{3}$ Communication and common randomness play the same role in this setting.
} 


\section{Public Channel}

What if the communication used for distributed channel synthesis occurs over a public channel and we wish for the synthesis to be immune to statistical tests that utilize the public message $J$ ? We require $X^{n}$ and $Y^{n}$ to pass as the input and output of a memoryless channel and $J$ to appear unrelated to $X^{n}$ and $Y^{n}$. That is, for rates $\left(R, R_{0}\right)$ to be achievable, there must exist a sequence of $\left(R, R_{0}\right)$ channel synthesis codes such that the induced distribution $P_{X^{n}, Y^{n}, J}$ satisfies

$$
\lim _{n \rightarrow \infty}\left\|P_{X^{n}, Y^{n}, J}-P_{J} \prod Q_{X} Q_{Y \mid X}\right\|_{T V}=0 \text {. }
$$

This setting falls into the context of secrecy, related to [27], [28], and [22]. Common randomness can be used as a secret key to encrypt the public communication. We find that this straightforward adaptation to distributed channel synthesis, where extra common randomness is used as a one-time-pad on the public communication, produces the optimal rate pairs $\left(R, R_{0}\right)$. The closure of the set of achievable rate pairs is given by

$$
\mathcal{S}_{P C}=\left\{\begin{aligned}
\left(R, R_{0}\right) \in \mathcal{R}^{2} & : \exists P_{X, Y, U} \in \mathcal{D} \text { s.t. } \\
R & \geq I(X ; U), \\
R_{0} & \geq I(X, Y ; U) .
\end{aligned}\right\},
$$

where $\mathcal{D}$ is defined in 9 .

Surprisingly, the common randomness rate requirement $R_{0}$ is greater than the communication rate requirement $R$ in the case of public communication. The common randomness rate can be reduced to the common information $C_{Q}(X ; Y)$, and the communication rate can be reduced to $I_{Q}(X ; Y)$, but the two extremes cannot be achieved simultaneously in general.

Proof of this result is in the appendix.

\section{Limited Duration Fidelity}

Consider a relaxed objective for channel synthesis. Suppose the objective is to synthesize a memoryless channel with high enough fidelity that it would pass any statistical test with limited memory of length $B$. That is, for any $\epsilon>0$ we desire an encoding such that

$$
\left\|P_{X_{t-B}^{t}, Y_{t-B}^{t}}-\prod Q_{X} Q_{Y \mid X}\right\|_{T V} \leq \epsilon \forall t
$$

where $B$ may be much smaller than the encoding block $n$.

The region of interest for a sharp rate requirement occurs when $B$ grows linearly with the encoding block-length: $B=$ $b n$. In this case, the region of achievable rate pairs $\left(R, R_{0}\right)$ contains the following region:

$$
\mathcal{S}_{L M}=\left\{\begin{aligned}
\left(R, R_{0}\right) \in \mathcal{R}^{2} & : \exists P_{X, Y, U} \in \mathcal{D} \text { s.t. } \\
R & \geq I(X ; U), \\
R_{0}+R & \geq b I(X, Y ; U) .
\end{aligned}\right\}
$$

where $\mathcal{D}$ is defined in 9 .

In particular this means that for finite memory $B$ not growing with $n$, no common randomness is required, and the communication rate must only exceed $R \geq I_{Q}(X ; Y)$. Notice that the sum-rate bound in (40) is dominated by the communication rate bound in (40) when $U=Y$ and $n$ is large enough that $I_{Q}(X ; Y)>\frac{B}{n} H_{Q}(Y)$.

See the appendix for the proof.

\section{E. Local Randomness}

The optimal encoder design for distributed channel synthesis, presented in $\$ \mathrm{~V}$, calls for randomization at the encoder and decoder. The randomization at the encoder is insignificant and perhaps even avoidable altogether. It is easy to show, for example, that $H_{P}\left(J \mid X^{n}, K\right)$ scales no more than linearly with $n$ at a rate close to the arbitrarily small excess rate $R-I(X ; U)$, where $U$ is the auxiliary random variable in the region $\mathcal{S}$ of $(8)$. On the other hand, the private randomization required by the decoder is much larger. The decoder of $\bar{V}$ locally synthesizes a memoryless channel according to $P_{Y \mid U}$ and applies the input $u^{n}(j, k)$ from the codebook to this synthesized channel.

Here we quantify explicitly the amount of local randomness required by the decoder, similar to [34] and [35]. Let $R_{L}$ be the rate of random bits $L \in\left[2^{n R_{L}}\right]$ available to the decoder only, and define the decoder as a deterministic function

$$
G: \mathcal{J} \times \mathcal{K} \times \mathcal{L} \rightarrow \mathcal{Y}^{n} .
$$

This is depicted in Fig. 9

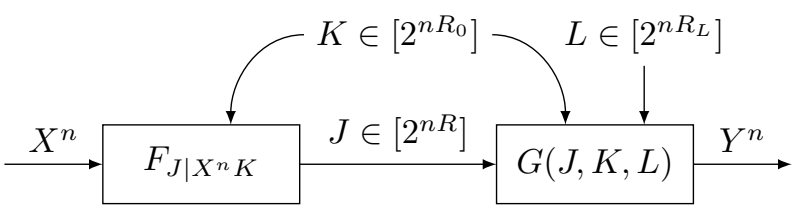

Fig. 9: Local Randomness: In this extension to the main result, the decoder is deterministic but makes use of rate-limited local randomness.

We now aim to characterize the set of rate triples $\left(R, R_{0}, R_{L}\right)$ that can synthesize a memoryless channel $Q_{Y \mid X}$ with input distribution $Q_{X}$, and we claim that the closure of this set is given by

$$
\mathcal{S}_{L R}=\left\{\begin{aligned}
\left(R, R_{0}, R_{L}\right) & \in \mathcal{R}^{3}: \\
\exists P_{X, Y, U} & \in \mathcal{D} \text { such that } \\
R & \geq I(X ; U), \\
R_{0}+R & \geq I(X, Y ; U), \\
R_{L} & \geq H(Y \mid U) .
\end{aligned}\right\},
$$

The total amount of randomness flowing into our synthetic channel (ignoring the minimally random encoder), when all inequalities in $\mathcal{S}_{L R}$ of (42) are exercised with equality, is $R_{0}+R_{L}=I(X, Y ; U)-I(X ; U)+H(Y \mid U)=H(Y \mid X)$. To our delight, distributed channel synthesis is efficient even compared to the local synthesis in [35] and in CorollaryVII.6.

This proof can be found in the appendix.

\section{F. Common Information Duality}

Two notions of common information were introduced at nearly the same time in the literature. One by Gács and Körner [5] is defined as

$$
C_{G-K}(X ; Y) \triangleq \max _{f(\cdot): H(f(X) \mid Y)=0} H(f(X)) .
$$

The other common information by Wyner in [6] is stated in (11). For this discussion, we refer to Wyner's common information as $C_{W}(X ; Y)$. 
Attention has been drawn in the literature to dual properties of these two quantities. For example,

$$
\begin{aligned}
C_{W}(X ; Y) & \geq I(X ; Y) \\
C_{G-K}(X ; Y) & \leq I(X ; Y)
\end{aligned}
$$

Also, both can be viewed as extreme points for the common message rate in the Gray-Wyner network [36]. In this network, correlated sources are encoded jointly using three messages and decoded separately, each decoder receiving only two of the messages. The message received by both is the common message. If we imagine the three messages traveling down a cable to a midway point (Segment 1) and then splitting into separate cables to travel to the separate decoders (Segment 2), with the common message duplicated at the juncture, then a simple duality can be stated. When the sum rate of the first segment is efficient, the common message rate is at least $C_{W}(X ; Y)$. When the sum rate of the second segment is efficient, the common message rate is no more than $C_{G-K}(X ; Y)$. Furthermore, the first case yields inefficiency in the second segment equal to $C_{W}(X ; Y)-I(X ; Y)$, and the second case yields inefficiency in the first segment equal to $I(X ; Y)-C_{G-K}(X ; Y)$. Thus, equality holds in both (44) and 45 or in neither.

Here we emphasize another duality, using the present results to enrich the operational symmetry.

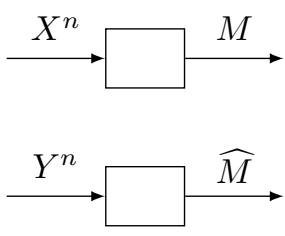

(a) Gács-Körner

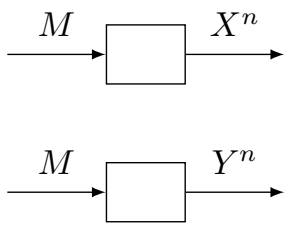

(b) Wyner
Fig. 10: Operational duality of common information.

Fig. 10 shows two complementary settings. In the first, i.i.d. observations of correlated random variables are used by separate, independent nodes to generate the same random bits (with high probability). The rate with which random bits can be generated is $C_{G-K}(X ; Y)$. In the second, equal random bits are provided to two independent nodes which must produce a correlated i.i.d. sequence (with high fidelity). The required rate of random bits is $C_{W}(X ; Y)$. These results come directly from the original work in [5] and [6].

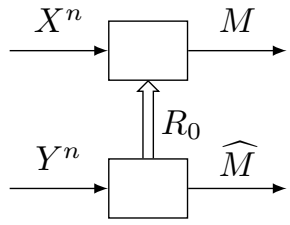

(a) Key Agreement

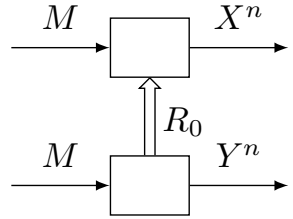

(b) Channel Synthesis
Fig. 11: Operational duality with communication: The rates of randomness $M$ for both situations relax to mutual information when communication is allowed (independent of the receiver output).
Now we add a communication link between the two encoders with a somewhat peculiar constraint. The communication is required to be independent of the output of the receiving node (nearly independent as measured by total variation). This alteration is depicted in Fig. 11

The setting of Fig. 11a has been studied for the purpose of secret key generation in [37], [38], [39], [40], and [41]. It is shown that with a high enough rate of communication, namely $H(Y \mid X)$, the rate of extraction of random bits in agreement increases to the mutual information $I(X ; Y)$.

The dual setting of Fig. 11b is solved by Theorem II.1 With a high enough communication rate, the required rate of random bits reduces to the mutual information $I(X ; Y)$. Furthermore, a communication rate of $H(Y \mid X)$ is sufficient in this setting as well (and necessary for most distributions).

To see how this follows from Theorem II.1 consider the equivalent description of the distributed channel synthesis problem given in the beginning of $₫ \mathrm{~V}$. That description applies exactly to this situation as well.

For added curiosity, we can state the corner points $\left(R, R_{0}\right)$ of the characterizations of the achievable rate region for the two settings in a way that suggests a deeper relationship. Both rate regions can be described as the union of simple regions, each defined by the choice of an auxiliary random variable. In the case of Fig. 11a the simple regions are rectangles defined by an upper bound on $R$ and a lower bound on $R_{0}$. In the case of Fig. $11 \mathrm{~b}$, the simple regions are pentagons defined by a lower bound on $R$ and a lower bound on the sum rate $\left(R+R_{0}\right)$. In both cases, we now specify the corner points that define the regions.

For the setting of Fig. 11a, the corner points of the rate region, identified in [39], are $\left(R, R_{0}\right)$ such that

$$
\begin{aligned}
R & =I(X ; U), \\
R_{0} & =I(Y ; U \mid X),
\end{aligned}
$$

for some $U$ such that $X-Y-U$ forms a Markov chain.

For the setting of Fig. $11 \mathrm{~b}$, the corner points of the rate region, identified by Theorem II.1 are $\left(R, R_{0}\right)$ such that

$$
\begin{aligned}
R & =I(X ; U), \\
R_{0} & =I(Y ; U \mid X),
\end{aligned}
$$

for some $U$ such that $X-U-Y$ forms a Markov chain.

\section{Soft Covering LEMmA}

\section{A. Discussion}

Our achievability proof centers around a soft covering lemmd 4 that is conceptually rooted in [6, Theorem 6.3] by Wyner and further studied in [17] and elsewhere. In this section we state the simple form of the lemma needed for our proof, accompanied by a new exponential bound. We then discuss this principle in greater depth, including proofs, in @II

The lemma pertains to the distribution induced by selecting uniformly at random from a random codebook and passing the codeword through a memoryless channel. If the size of

\footnotetext{
${ }^{4}$ In [23] we referred to this as the "cloud mixing" lemma.
} 
the codebook is large enough, then the resulting distribution on the output of the channel, illustrated in Fig. 12, will be well approximated by an i.i.d. distribution. Not surprisingly, the rate of the codebook sufficient to observe this phenomenon is the mutual information associated with the codebook distribution and the channel. Wyner used this observation in his achievability proof for common information. This lemma will also play a key role in our achievability proof for distributed channel synthesis, providing us with a simple analysis.

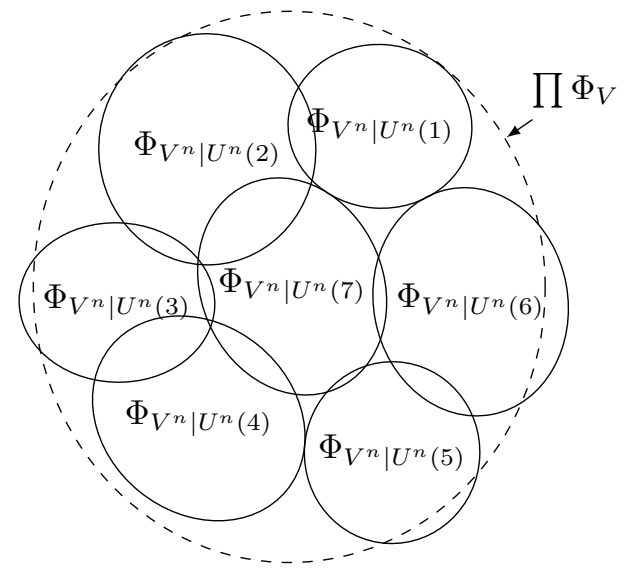

Fig. 12: Soft Covering: A sparse collection (codebook) of conditional distributions $\Phi_{V^{n} \mid U^{n}=u^{n}(i)}$ is averaged together to approximate a marginal distribution $\prod \Phi_{V}$. For an i.i.d. codebook distribution and a memoryless channel $\Phi_{V^{n} \mid U^{n}}$, an exponentially large codebook of rate $R>I(U ; V)$ is sufficient.

This phenomenon has a close relative, often referred to simply as covering. Given a joint distribution $P_{U, V}$, in the limit of large block-length, a random codebook of size $2^{n R}$ of $u^{n}$ sequences, with $R>I(U ; V)$, covers the i.i.d. distribution associated with $P_{V}$ in the sense that for a random $V^{n}$ there will exist with high probability a sequence in the codebook such that $\left(u^{n}, V^{n}\right)$ are jointly typical per the definition (10.107) in [32]. That is, the union of conditionally typical sets in $\mathcal{V}^{n}$ induced by the codebook will contain all of the probability of the i.i.d. distribution in the limit. The soft covering lemma is a strengthening of this statement. Instead of looking at the union of conditionally typical sets, it states that the average of conditional distributions from each codeword in the codebook will match the i.i.d. distribution to arbitrarily high fidelity.

In [17], Han and Verdú study this soft covering phenomenon in depth. Notably, they examine the converse statement, characterizing the necessary codebook rates, in addition to sufficient rates. This they refer to as the "resolvability" of a channel. Furthermore, their work looks beyond memoryless channels. Also, they consider total variation as a metric for the fidelity of the distribution (as we do here), in addition to normalized K-L divergence, which was the metric that Wyner used.

Other work has also continued the study of this phenomenon. Hayashi's derivation in [42] provided the tightest previous bound in the literature for memoryless channels, upon which this work improves. Also, [43] and Chapter 16 of [44] contain similar lemmas in a broader quantum context, both referring to the tool as a "covering lemma." The "sampling lemma" in [26] makes a particularly strong claim that the soft covering phenomenon occurs even under the stricter fidelity metric of K-L divergence (not normalized by the block-length).

Recent work in [19] and [45] has developed alternative constructions and analysis tools for obtaining similar properties to what soft covering provides, partially motivated by our work in [1] and [2].

\section{B. Soft Covering Lemma Statement}

The simplest statement of the soft covering principle, and all that we need for the proof of distributed channel synthesis, involves a memoryless channel with memoryless input. Let $\Phi_{U}$ be a distribution on $\mathcal{U}$ that induces a distribution $\Phi_{V}$ when applied to the channel $\Phi_{V \mid U}$. For $n$ channel uses, the corresponding input-output joint distribution is then

$$
\begin{aligned}
& \Phi_{U^{n}, V^{n}}=\quad \begin{array}{cc}
\text { Input } & \text { Channel } \\
\left(\prod \Phi_{U}\right) & \left(\prod \Phi_{V \mid U}\right)
\end{array} \\
& =\prod \Phi_{U, V}
\end{aligned}
$$

yielding the desired output distribution

$$
\begin{aligned}
Q_{V^{n}}\left(v^{n}\right) & \triangleq \sum_{u^{n}} \Phi_{U^{n}, V^{n}}\left(u^{n}, v^{n}\right) \\
& =\prod_{t=1}^{n} \Phi_{V}\left(v_{t}\right) .
\end{aligned}
$$

The lemma, which follows, states that we can nearly produce the desired output distribution by applying a uniform distribution to a collection $\mathcal{B}^{(n)}$ of $2^{n(I(U ; V)+\epsilon)}$ randomly generated channel input sequences, as depicted in Fig. 13. The criterion for nearly producing the desired output distribution is that the induced output distribution

$$
P_{V^{n}}\left(v^{n}\right) \triangleq \frac{1}{\left|\mathcal{B}^{(n)}\right|} \sum_{j=1}^{\left|\mathcal{B}^{(n)}\right|} \Phi_{V^{n} \mid U^{n}}\left(v^{n} \mid u^{n}(j)\right)
$$

has vanishing total variation from the desired output distribution as $n$ increases.

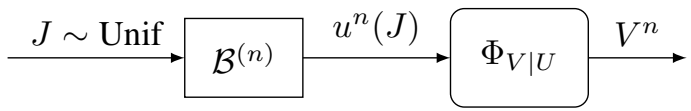

Fig. 13: Soft covering - Memoryless channel: An i.i.d. output distribution $\prod \Phi_{V}$ is synthesized by randomly selecting a codeword from a codebook of $u^{n}$ sequences and passing it through a memoryless channel. Lemma IV.1 gives a sufficient codebook construction.

Lemma IV.1 (Soft Covering - Memoryless channel). Let $\mathcal{B}^{(n)}$ be a randomly generated collection of $2^{n R}$ sequences in $\mathcal{U}^{n}$, each drawn independently and i.i.d. from the codebook distribution $\Phi_{U}$. A memoryless channel specified by $\Phi_{V \mid U}$ induces an output distribution defined in (54). This output distribution is random because $\mathcal{B}^{(n)}$ is random. 
If $R>I_{\Phi}(U ; V)$ then the expected value of the total variation between the induced output distribution and the desired output distribution defined in (52) vanishes with $n$. That is,

$$
R>I_{\Phi}(U ; V) \Longrightarrow \lim _{n \rightarrow \infty} \mathbf{E}\left\|P_{V^{n}}-Q_{V^{n}}\right\|_{T V}=0 .
$$

Furthermore, the expected total variation vanishes exponentially fast:

$$
\mathbf{E}\left\|P_{V^{n}}-Q_{V^{n}}\right\|_{T V} \leq \frac{3}{2} \exp (-\gamma n),
$$

where $\gamma$ is given in (126) of $₫ V I I-D$

LemmaIV.1 can be derived as a corollary of Theorem VII.1 which is stated in $\$ \mathrm{VII}$ as a generalization of the soft covering principle, along with a variety of corollaries, the proof, and analysis of the total variation exponent.

\section{ACHIEVABILITy}

\section{A. Synopsis}

In this section we prove $\mathcal{C} \supset \mathcal{S}$. That is to say, for any rate pair $\left(R, R_{0}\right)$ in the interior of the rate region specified by $\mathcal{S}$, $\left(R, R_{0}\right)$ is achievable for synthesizing the memoryless channel $Q_{Y \mid X}$ with input distribution $Q_{X}$. The definition of achievability in Definition 5 concerns the existence of $\left(R, R_{0}, n\right)$ channel synthesis codes. However, the same achievability criterion can be stated simply in terms of the existence of a joint distribution satisfying certain properties, removing the emphasis from the usual causal description of how an encoder or decoder takes an input and returns an output. This method can be used to redefine any of the familiar communication problems in information theory, but we find it particularly useful in this case.

Consider the induced joint distribution $P_{X^{n}, Y^{n}, J, K}$ defined in Definition 3 . The rates $\left(R, R_{0}\right)$ are achievable if for any $\epsilon>0$ there exists an $N$ such that for all block lengths $n>N$ there exists an induced joint distribution $P_{X^{n}, Y^{n}, J, K}$ satisfying the following properties:

1) $X^{n}-(J, K)-Y^{n}$ form a Markov chain.

2) $X^{n}$ and $K$ are independent.

3) $X^{n}$ is i.i.d. $\sim Q_{X}$.

4) $|\mathcal{J}|=2^{n R}$.

5) $|\mathcal{K}|=2^{n R_{0}} \cdot 5$

6) $\left\|P_{X^{n}, Y^{n}}-\prod Q_{X} Q_{Y \mid X}\right\|_{T V}<\epsilon$.

This is simply an exhaustive list of all of the constraints imposed by the definitions of channel synthesis codes and the induced joint distribution, with the addition of 6), the synthesis requirement.

Our approach will be to construct a joint distribution $\Upsilon_{X^{n}, Y^{n}, J, K}$ that satisfies 1), 4), and 5) by construction. We will then use the soft covering lemma of $₫[$ to show that 6 ) is satisfied while 2) and 3) are nearly satisfied. Fortunately, due to some basic properties of total variation, we can augment the joint distribution to exactly satisfy 2) and 3) while not destroying the other properties.

\footnotetext{
${ }^{5}$ The result does not change if $K$ is required to be uniformly distributed per the original problem statement.
}

The key idea for developing this proof is to relax some of the strict requirements (properties 2) and 3)), knowing that this relaxation can be corrected at the end. By doing so, we reveal a large degree of symmetry in the problem statement. Rather than design the joint distribution from left to right (referring to the Markov chain in property 1)), we design from the middle outward.

The consequence of this technique is that we design the encoder in reverse. The result is best described as a likelihood encoder (see $₫ \mathrm{~V}$-D), which is stochastic. Similarity between the behavior of this encoder and other encoders used for source coding is analyzed in [45]. Also, an alternative proof construction based on random binning, which yields similar behavior to the likelihood encoder, is proposed in [19].

\section{B. Construction}

Begin by finding $Q_{X, Y, U} \in \mathcal{D}$ defined in (9) such that $R>I_{Q}(X ; U)$ and $R_{0}+R>I_{Q}(X, Y ; U)$. Our reuse of the label $Q$ is intentional. By the definition of $\mathcal{D}$, the marginal distribution of $Q_{X, Y, U}$ must coincide with the desired inputoutput distribution specified by $Q_{X} Q_{Y \mid X}$.

Using the standard practice of random codebook construction to prove the existence of a good codebook, generate a codebook $\mathcal{B}^{(n)}$ of $u^{n}$ sequences indexed by $j \in\left[2^{n R}\right]$ and $k \in\left[2^{n R_{0}}\right]$ independently according to $\prod_{t=1}^{n} Q_{U}\left(u_{t}\right)$. Construct a joint distribution as depicted in Fig. 14 and as follows. Define $\Upsilon_{X^{n}, Y^{n}, J, K}$ such that $J$ and $K$ are uniformly distributed over their supports and $X^{n}$ and $Y^{n}$ are the result of the codeword $u^{n}(J, K)$ passed through the memoryless channel defined by $Q_{X, Y \mid U}$ :

$$
\begin{aligned}
& \Upsilon_{X^{n}, Y^{n}, J, K}\left(x^{n}, y^{n}, j, k\right) \\
& \triangleq \frac{1}{2^{n\left(R_{0}+R\right)}}\left(\prod_{t=1}^{n} Q_{X, Y \mid U}\left(x_{t}, y_{t} \mid u_{t}(j, k)\right)\right) .
\end{aligned}
$$

Notice that the channel $Q_{X, Y \mid U}$ separates into $Q_{X \mid U} Q_{Y \mid U}$, as shown in Fig. 14 because of the Markov chain property of all distributions in $\mathcal{D}$.

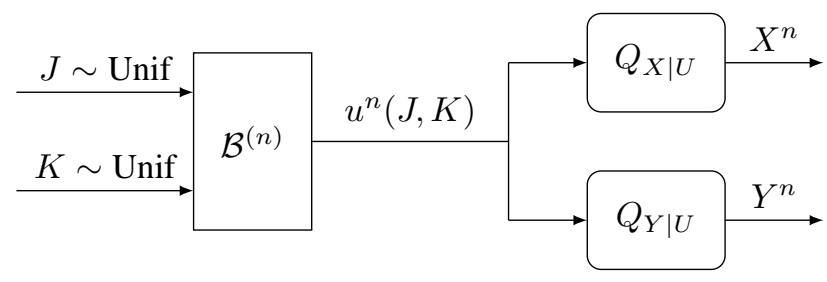

Fig. 14: Codec Construction: The first step in deriving an efficient encoder and decoder is to construct a joint distribution $\Upsilon_{X^{n}, Y^{n}, J, K}$ that nearly satisfies the six conditions for achievability. This is done by constructing a randomly generated codebook $\mathcal{B}^{(n)}$ of sequences $u^{n}(j, k)$. Independent and uniformly distributed indices $J$ and $K$ select from the codebook the input to a memoryless broadcast channel specified by $Q_{X \mid U} Q_{Y \mid U}$. If the cardinalities of $J$ and $K$ are large enough, $X^{n}$ and $Y^{n}$ can be shown using the soft covering lemma to be nearly i.i.d. according to the desired distribution, with $X^{n}$ nearly independent of $K$. 
It is clear that $\Upsilon_{X^{n}, Y^{n}, J, K}$ satisfies properties 1), 4), and $5)$ by construction. Our next step is to construct $P_{X^{n}, Y^{n}, J, K}$ from $\Upsilon_{X^{n}, Y^{n}, J, K}$ in a way that satisfies properties 2) and 3 ). Define $P_{X^{n}, Y^{n}, J, K}$ as follows:

$$
P_{X^{n}, Y^{n}, J, K}=\frac{1}{2^{n R_{0}}}\left(\prod Q_{X}\right) \Upsilon_{Y^{n}, J \mid X^{n}, K} .
$$

The conditional distribution $\Upsilon_{Y^{n}, J \mid X^{n}, K}$ is derived from $\Upsilon_{X^{n}, Y^{n}, J, K}$ and well defined for all values of $\left(X^{n}, K\right)$ with positive probability. For others values we can simply assign the uniform distribution over $\mathcal{Y}^{n} \times \mathcal{J}$.

Notice that $P_{X^{n}, Y^{n}, J, K}$ satisfies property 1) because $P_{Y^{n} \mid X^{n}, J, K}=\Upsilon_{Y^{n} \mid X^{n}, J, K}=\Upsilon_{Y^{n} \mid J, K}$. Thus, $\Upsilon_{J \mid X^{n}, K}$ defines the likelihood encoder, and $\Upsilon_{Y^{n} \mid J, K}$ is the decoder. The distribution $P_{X^{n}, Y^{n}, J, K}$ satisfies 2), 3), 4), and 5) as well by construction. Only property 6 ) is left to be verified.

\section{Synthesis Analysis}

Recall that $\Upsilon_{X^{n}, Y^{n}, J, K}$ and $P_{X^{n}, Y^{n}, J, K}$ are random because the codebook $\mathcal{B}^{(n)}$ is random. We now call on the soft covering lemma (Lemma IV.1) twice. First, we have a straightforward conclusion. Since $R_{0}+R>I_{Q}(X, Y ; U)$,

$$
\lim _{n \rightarrow \infty} \mathbf{E}\left\|\Upsilon_{X^{n}, Y^{n}}-\prod Q_{X} Q_{Y \mid X}\right\|_{T V}=0
$$

The second use of Lemma IV.1 is a little more subtle and similar to a technique used by Winter in [26] and by Bloch and Laneman in [31]. Notice that for any fixed $k$, the collection $\left\{u^{n}(j, k)\right\}_{j}$ is a collection of $2^{n R}$ randomly generated codewords. If we consider only the memoryless channel specified by $Q_{X \mid U}$ with channel output $X^{n}$, then $R>I_{Q}(U ; X)$ satisfies the condition of the lemma. Therefore, for any $k$,

$$
\mathbf{E}\left\|\Upsilon_{X^{n} \mid K=k}-\prod Q_{X}\right\|_{T V}<\epsilon_{n} \rightarrow 0
$$

as $n \rightarrow \infty$. The expression on the left-hand side is constant over all values of $k$ for any fixed $n$ because of the symmetric nature of an i.i.d. codebook.

By the definition of total variation in (26),

$$
\begin{aligned}
\mathbf{E} & \left\|\Upsilon_{X^{n}, K}-\frac{1}{2^{n R_{0}}} \prod Q_{X}\right\|_{T V} \\
& =\mathbf{E} \frac{1}{2} \sum_{x^{n}, k}\left|\Upsilon_{X^{n}, K}\left(x^{n}, k\right)-\frac{1}{2^{n R_{0}}} \prod_{t=1}^{n} Q_{X}\left(x_{t}\right)\right| \\
& =\frac{1}{2^{n R_{0}}} \mathbf{E} \frac{1}{2} \sum_{x^{n}, k}\left|\Upsilon_{X^{n} \mid K}\left(x^{n} \mid k\right)-\prod_{t=1}^{n} Q_{X}\left(x_{t}\right)\right| \\
& =\frac{1}{2^{n R_{0}}} \sum_{k} \mathbf{E} \| \Upsilon_{X^{n} \mid K=k}-\left.\prod Q_{X}\right|_{T V} \\
& \leq \frac{1}{2^{n R_{0}}} \sum_{k} \epsilon_{n} \\
& =\epsilon_{n} \\
& \rightarrow 0 .
\end{aligned}
$$

Thus, $\Upsilon_{X^{n}, Y^{n}, J, K}$ satisfies property 6) by (59), and it nearly satisfies properties 2) and 3) by (61). We next invoke two properties of total variation.
Lemma V.1 (Total Variation of Marginal Distribution). Total variation cannot be larger between marginal distributions than between encompassing joint distributions. That is,

$$
\left\|\Pi_{W}-\Gamma_{W}\right\|_{T V} \leq\left\|\Pi_{W, Z}-\Gamma_{W, Z}\right\|_{T V} .
$$

Proof: Referring to the definition of total variation given in (23), the left-hand side of (62) is a maximization over a smaller set than the right-hand side.

Lemma V.2 (Total Variation with Common Channel). When two random variables are passed through the same channel, the total variation between the resulting input-output joint distributions is the same as the total variation between the input distributions. That is,

$$
\left\|\Pi_{W} \Pi_{Z \mid W}-\Gamma_{W} \Pi_{Z \mid W}\right\|_{T V}=\left\|\Pi_{W}-\Gamma_{W}\right\|_{T V} .
$$

Proof: Referring to the equivalent definition for total variation given in (25), the non-negative $\Pi_{Z \mid W}$ term from the left-hand side of (63) factors out of the absolute value and sums to one.

We continue with the final steps of the analysis of $P_{X^{n}, Y^{n}}$ using the triangle inequality:

$$
\begin{aligned}
& \left\|P_{X^{n}, Y^{n}}-\prod Q_{X} Q_{Y \mid X}\right\|_{T V} \\
& \leq\left\|P_{X^{n}, Y^{n}}-\Upsilon_{X^{n}, Y^{n}}\right\|_{T V} \\
& +\left\|\Upsilon_{X^{n}, Y^{n}}-\prod Q_{X} Q_{Y \mid X}\right\|_{T V} \\
& \stackrel{(a)}{\leq}\left\|P_{X^{n}, Y^{n}, J, K}-\Upsilon_{X^{n}, Y^{n}, J, K}\right\|_{T V} \\
& +\left\|\Upsilon_{X^{n}, Y^{n}}-\prod Q_{X} Q_{Y \mid X}\right\|_{T V} \\
& \stackrel{(b)}{=}\left\|P_{X^{n}, K}-\Upsilon_{X^{n}, K}\right\|_{T V} \\
& +\left\|\Upsilon_{X^{n}, Y^{n}}-\prod Q_{X} Q_{Y \mid X}\right\|_{T V} \\
& =\left\|\frac{1}{2^{n R_{0}}}\left(\prod Q_{X}\right)-\Upsilon_{X^{n}, K}\right\|_{T V} \\
& +\left\|\Upsilon_{X^{n}, Y^{n}}-\prod Q_{X} Q_{Y \mid X}\right\|_{T V} .
\end{aligned}
$$

Both terms vanish as $n \rightarrow \infty$ because of (61) and (59). Inequality (a) comes from Lemma V.1 and (b) comes from Lemma V.2

Therefore, for $n$ large enough, there exists a distribution satisfying all properties for achievability. Furthermore, the soft covering lemma asserts that each of the total variation terms in the bound vanishes exponentially quickly.

\section{Comments}

We now summarize the behavior of the optimal encoder and decoder constructed in this section:

Likelihood Encoder: The encoder inspects the codebook $\mathcal{B}^{(n)}$ of $u^{n}$ sequences indexed by $j$ and $k$ and considers only the subset where $k$ is equal to the common randomness observed. In other words, the common randomness selects a sub-codebook. The encoder then considers each $u^{n}$ sequence in the sub-codebook and selects one randomly with probability 
proportional to its likelihood associated with the memoryless channel $Q_{X \mid U}$ and the observed source sequence $X^{n}$.

It may happen that every codeword has a positive probability of being selected by the encoder; however, most of the probability will be concentrated on those codewords that are jointly typical with $X^{n}$. Still, there are many jointly typical sequences to choose from randomly. An interesting endeavor would be to design a deterministic encoder, if possible, that successfully operates throughout the region where both inequalities of Theorem II.1 are active.

Decoder: The decoder identifies a codeword $u^{n}$ given by the codebook $\mathcal{B}^{(n)}$, the common randomness $K$, and the message $J$. He then locally synthesizes a memoryless channel according to $Q_{Y \mid U}$ to produce $Y^{n}$ from $u^{n}$.

In the decoder's case, a specific amount of randomization ( $H(Y \mid U)$ per channel use) is fundamental to the design and unavoidable according to

\section{CONVERSE}

In this section we prove $\mathcal{C} \subset \mathcal{S}$. That is, any achievable rate pair $\left(R, R_{0}\right)$ for synthesizing the memoryless channel $Q_{Y \mid X}$ with input distribution $Q_{X}$ must fall in $\mathcal{S} 6$

\section{A. Cardinality Bound}

The cardinality bound on the auxiliary random variable $U$ in the definition of $\mathcal{D}$ in $(9)$ not only makes the region computable but is an essential step in the converse, as will be apparent in 乌I-D

Lemma VI.1 (Cardinality Bound). For any discrete random variables $(X, Y, W) \sim \Pi_{X, Y, W}$ forming a Markov chain $X-$ $W-Y$, there exists a distribution $\Gamma_{X, Y, U}$ forming a Markov chain $X-U-Y$ such that

$$
\begin{aligned}
|\mathcal{U}| & \leq|\mathcal{X}||\mathcal{Y}|+1, \\
\Gamma_{X, Y} & =\Pi_{X, Y}, \\
I_{\Gamma}(X ; U) & =I_{\Pi}(X ; W), \\
I_{\Gamma}(X, Y ; U) & =I_{\Pi}(X, Y ; W) .
\end{aligned}
$$

Proof: Consider the set of points $\mathcal{A} \in \mathcal{R}^{|\mathcal{X}||\mathcal{Y}|+2}$ such that the first $|\mathcal{X}||\mathcal{Y}|$ coordinates represent the mass values of a product (independent) distribution $P_{X} P_{Y}$ and the last two coordinates are $H_{P}(X)$ and $H_{P}(X, Y)$. This is a connected and compact set because each coordinate is a continuous function on the connected and compact set of all product distributions.

Recall that $\Pi_{X, Y, W}$ is the distribution of the Markov chain $X-W-Y$ in question. Consider the point $\pi \in$ $\mathcal{R}^{|\mathcal{X}||\mathcal{Y}|+2}$ where $\Pi_{X, Y}$ specifies the first $|\mathcal{X}||\mathcal{Y}|$ coordinates and $H_{\Pi}(X \mid W)$ and $H_{\Pi}(X, Y \mid W)$ the last two. Notice that $\pi$ is in the convex hull of $\mathcal{A}$. It is a convex combination of points, each represented by a particular value of $w$, with convex weight equal to $\Pi_{W}(w)$. The constituent product distributions are the distributions of $(X, Y)$ conditioned on $W=w$.

\footnotetext{
${ }^{6}$ The set $\mathcal{S}$ is a closed set.
}

Notice that the connected and compact set $\mathcal{A}$ is in fact contained in a $(|\mathcal{X}||\mathcal{Y}|+1)$-dimensional subspace of $\mathcal{R}^{|\mathcal{X}||\mathcal{Y}|+2}$ because of the linear constraint that a probability mass function sum to one. Thus, the Carathéodory theorem for a connected set 7 states that $\pi$ is a convex combination of $(|\mathcal{X}||\mathcal{Y}|+1)$ points in $\mathcal{A}$ (see original publications: [46] for compact sets, [47] for general sets, [48] for connected sets; application to cardinality bounds of auxiliary variables: [49], [50, Lemma 15.4], [51]). Associate each point with a value $u$. We use these points to construct the distribution $\Gamma_{X, Y, U}$. The convex weight of the points becomes $\Gamma_{U}(u)$, and the associated product distributions are the conditional distributions $\Gamma_{X, Y \mid U=u}$, yielding the desired Markov chain property $X-U-Y$. Notice that the joint distribution of $(X, Y)$ and the conditional entropies are preserved by the construction of $\pi$.

\section{B. Entropy bounds}

A few preliminary bounds are needed to show that sequences that are nearly i.i.d. in total variation will have information properties close to their i.i.d. counterparts.

Lemma VI.2 (Total Variation of Random Sample). The total variation between the distributions of two random sequences is an upper bound on the total variation between the distributions of the variables in the sequences at a random time index (independent of the sequences).

Let $T \in\{1, \ldots, n\}$ be a random time index distributed according to $\Pi_{T}$. Also let $\Pi_{W^{n}}$ and $\Gamma_{W^{n}}$ be distributions independent of $T$, so that $\Pi_{W^{n}, T}=\Pi_{W^{n}} \Pi_{T}$ and $\Gamma_{W^{n}, T}=$ $\Gamma_{W^{n}} \Pi_{T}$. Then,

$$
\left\|\Pi_{W_{T}}-\Gamma_{W_{T}}\right\|_{T V} \leq\left\|\Pi_{W^{n}}-\Gamma_{W^{n}}\right\|_{T V} .
$$

Proof: Notice that the channel $\Pi_{W_{T} \mid W^{n}}\left(a \mid w^{n}\right)=$ $\sum_{t=1}^{n} \Pi_{T}(t) \mathbf{1}\left(a=w_{t}\right)$ defines the process that takes $W^{n}$ and selects a random time index according to $\Pi_{T}$. This Lemma simply requires that output distributions from a common channel are as close as input distributions in total variation-a consequence of Lemma V.1 and Lemma V.2.

Now we build on the fact that for finite alphabets we can upper-bound the difference in entropy in terms of total variation [32, Theorem 17.3.3].

Lemma VI.3 (Entropy and Timing Information of Nearly i.i.d. Sequences). For any discrete random sequence $W^{n} \sim \Pi_{W^{n}}$ where $W_{t} \in \mathcal{W}$ for all $t \in\{1, \ldots, n\}$, if there exists a distribution $\Gamma_{W}$ on the alphabet $\mathcal{W}$ such that

$$
\left\|\Pi_{W^{n}}-\prod \Gamma_{W}\right\|_{T V} \leq \epsilon<1 / 4
$$

then

$$
\frac{1}{n} \sum_{t=1}^{n} I_{\Pi}\left(W_{t} ; W^{t-1}\right) \leq 4 \epsilon\left(\log |\mathcal{W}|+\log \frac{1}{\epsilon}\right),
$$

and for any random variable $T \in\{1, \ldots, n\}$ independent of $W^{n}$,

$$
I_{\Pi}\left(W_{T} ; T\right) \leq 4 \epsilon\left(\log |\mathcal{W}|+\log \frac{1}{\epsilon}\right) .
$$

\footnotetext{
${ }^{7}$ This theorem is often referred to as the Carathéodory-Fenchel-Eggleston theorem.
} 
Proof: We start by applying LemmaVI.2 for the arbitrary random time index $T$ referred to in the Lemma as well as for each individual deterministic time index (each a special case of $\left.\Pi_{T}\right)$. Then by Theorem 17.3.3 of [32],

$$
\begin{aligned}
\left|H_{\Pi}\left(W^{n}\right)-H_{\Gamma}\left(W^{n}\right)\right| & \leq 2 \epsilon \log \left(\frac{|\mathcal{W}|^{n}}{\epsilon}\right), \\
\left|H_{\Pi}\left(W_{T}\right)-H_{\Gamma}(W)\right| & \leq 2 \epsilon \log \left(\frac{|\mathcal{W}|}{\epsilon}\right), \\
\left|H_{\Pi}\left(W_{t}\right)-H_{\Gamma}(W)\right| & \leq 2 \epsilon \log \left(\frac{|\mathcal{W}|}{\epsilon}\right),
\end{aligned}
$$

for all $t \in\{1, \ldots, n\}$.

As with any i.i.d. distribution, $H_{\Gamma}\left(W^{n}\right)=\sum_{t=1}^{n} H_{\Gamma}\left(W_{t}\right)$. Therefore, the triangle inequality yields,

$$
\begin{aligned}
& \frac{1}{n} \sum_{t=1}^{n} I_{\Pi}\left(W_{t} ; W^{t-1}\right) \\
& =\frac{1}{n}\left(\left(\sum_{t=1}^{n} H_{\Pi}\left(W_{t}\right)\right)-H_{\Pi}\left(W^{n}\right)\right) \\
& \leq \frac{1}{n}\left|H_{\Pi}\left(W^{n}\right)-H_{\Gamma}\left(W^{n}\right)\right| \\
& \quad+\frac{1}{n} \sum_{t=1}^{n}\left|H_{\Pi}\left(W_{t}\right)-H_{\Gamma}(W)\right| \\
& \stackrel{(a)}{\leq} \frac{2}{n} \epsilon \log \left(\frac{|\mathcal{W}|^{n}}{\epsilon}\right)+2 \epsilon \log \left(\frac{|\mathcal{W}|}{\epsilon}\right) \\
& =4 \epsilon \log |\mathcal{W}|+\frac{n+1}{n} 2 \epsilon \log \frac{1}{\epsilon} \\
& \leq 4 \epsilon\left(\log |\mathcal{W}|+\log \frac{1}{\epsilon}\right),
\end{aligned}
$$

where (a) refers to (73) and (75).

Furthermore, denoting the distribution of $T$ as $\Pi_{T}$, notice that

$$
H_{\Pi}\left(W_{T} \mid T\right)=\sum_{t=1}^{n} \Pi_{T}(t) H_{\Pi}\left(W_{t}\right)
$$

because of the independence of $T$ and $W^{n}$. Therefore,

$$
\begin{aligned}
\left|H_{\Pi}\left(W_{T} \mid T\right)-H_{\Gamma}(W)\right| & \mid \\
& =\left|\sum_{t=1}^{n} \Pi_{T}(t)\left(H_{\Pi}\left(W_{t}\right)-H_{\Gamma}(W)\right)\right| \\
& \leq \sum_{t=1}^{n} \Pi_{T}(t)\left|H_{\Pi}\left(W_{t}\right)-H_{\Gamma}(W)\right| \\
& \stackrel{(a)}{\leq} \sum_{t=1}^{n} \Pi_{T}(t) 2 \epsilon \log \left(\frac{|\mathcal{W}|}{\epsilon}\right) \\
& =2 \epsilon \log \left(\frac{|\mathcal{W}|}{\epsilon}\right),
\end{aligned}
$$

where (a) refers to (75). Combining this with (74) gives

$$
I_{\Pi}\left(W_{T} ; T\right) \leq 4 \epsilon\left(\log |\mathcal{W}|+\log \frac{1}{\epsilon}\right)
$$

by way of the triangle inequality.

\section{Epsilon Rate Region}

Now we use information theoretic inequalities and lemmas VI.3 and VI.1 to nearly complete the proof. We define a region $\mathcal{S}_{\epsilon}$ for $\epsilon>0$ that gracefully expands the region $\mathcal{S}$ of the main result. Then we show that an achievable rate pair $\left(R, R_{0}\right)$ is in $\mathcal{S}_{\epsilon}$.

Let the epsilon rate region be defined as

$$
\mathcal{S}_{\epsilon} \triangleq\left\{\begin{aligned}
\left(R, R_{0}\right) & \in \mathcal{R}^{2}: \\
\exists P_{X, Y, U} & \in \mathcal{D}_{\epsilon} \text { such that } \\
R & \geq I(X ; U), \\
R_{0}+R & \geq I(X, Y ; U)-2 g(\epsilon) .
\end{aligned}\right\},
$$

where

$$
\mathcal{D}_{\epsilon} \triangleq\left\{\begin{array}{l}
P_{X, Y, U}: \\
\left\|P_{X, Y}-Q_{X} Q_{Y \mid X}\right\|_{T V} \leq \epsilon \\
X-U-Y \text { Markov, } \\
|\mathcal{U}| \leq|\mathcal{X}||\mathcal{Y}|+1
\end{array}\right\}
$$

and

$$
g(\epsilon) \triangleq 4 \epsilon\left(\log |\mathcal{X}|+\log |\mathcal{Y}|+\log \frac{1}{\epsilon}\right) .
$$

Lemma VI.4 (Epsilon Rate Region). If the rate pair $\left(R, R_{0}\right)$ is achievable for channel $Q_{Y \mid X}$ and source $Q_{X}$, then

$$
\left(R, R_{0}\right) \in \mathcal{S}_{\epsilon} \quad \forall \epsilon>0 .
$$

Proof: Since $\mathcal{S}_{\epsilon}$ shrinks with $\epsilon$, let us only consider $\epsilon<1 / 4$. Let $\left(R, R_{0}\right)$ be achievable. Then there exists an $\left(R, R_{0}, n\right)$ channel synthesis code such that

$$
\left\|P_{X^{n}, Y^{n}}-\prod Q_{X} Q_{Y \mid X}\right\|_{T V}<\epsilon .
$$

Let the random variable $T$ be uniformly distributed over the set $\{1, \ldots, n\}$ and independent of the induced joint distribution $P_{X^{n}, Y^{n}, J, K}$. The variable $T$ will serve as a random time index. The variable $X_{T}$ is independent of $T$ because $X^{n}$ is an i.i.d. source sequence (see [2], Property 1). However, $Y_{T}$ need not be independent of $T$.

We lower bound $R$ by,

$$
\begin{aligned}
n R & \geq H_{P}(J) \\
& \geq H_{P}(J \mid K) \\
& \geq I_{P}\left(X^{n} ; J \mid K\right) \\
& \stackrel{(a)}{=} I_{P}\left(X^{n} ; J, K\right) \\
& =\sum_{t=1}^{n} I_{P}\left(X_{t} ; J, K \mid X^{t-1}\right) \\
& \stackrel{(b)}{=} \sum_{t=1}^{n} I_{P}\left(X_{t} ; J, K, X^{t-1}\right) \\
& \geq \sum_{t=1}^{n} I_{P}\left(X_{t} ; J, K\right) \\
& =n I_{P}\left(X_{T} ; J, K \mid T\right) \\
& \stackrel{(c)}{=} n I_{P}\left(X_{T} ; J, K, T\right),
\end{aligned}
$$

where (a) comes from the problem statement and (b) and (c) are due to the i.i.d. nature of $X^{n}$. 
Similarly, we lower bound the sum rate by,

$$
\begin{aligned}
& n\left(R_{0}+R\right) \\
& \quad \geq H_{P}(J, K) \\
& \geq I_{P}\left(X^{n}, Y^{n} ; J, K\right) \\
& \quad=\sum_{t=1}^{n} I_{P}\left(X_{t}, Y_{t} ; J, K \mid X^{t-1}, Y^{t-1}\right) \\
& \quad=\sum_{t=1}^{n} I_{P}\left(X_{t}, Y_{t} ; J, K, X^{t-1}, Y^{t-1}\right) \\
& \quad \quad-\sum_{t=1}^{n} I_{P}\left(X_{t}, Y_{t} ; X^{t-1}, Y^{t-1}\right) \\
& \quad \stackrel{(a)}{\geq} \sum_{t=1}^{n} I_{P}\left(X_{t}, Y_{t} ; J, K, X^{t-1}, Y^{t-1}\right)-n g(\epsilon) \\
& \geq \sum_{t=1}^{n} I_{P}\left(X_{t}, Y_{t} ; J, K\right)-n g(\epsilon) \\
& \quad=n I_{P}\left(X_{T}, Y_{T} ; J, K \mid T\right)-n g(\epsilon) \\
& \quad=n I_{P}\left(X_{T}, Y_{T} ; J, K, T\right)-n I_{P}\left(X_{T}, Y_{T} ; T\right)-n g(\epsilon) \\
& \stackrel{(b)}{\geq} n I_{P}\left(X_{T}, Y_{T} ; J, K, T\right)-2 n g(\epsilon),
\end{aligned}
$$

where (a) and (b) are both consequences of LemmaVI.3, and $g(\epsilon)$ is defined in (82).

Notice the Markov chain given by $X_{T}-(J, K, T)-Y_{T}$. This comes about because the entire sequences $X^{n}$ and $Y^{n}$ are conditionally independent given $J$ and $K$, according to the problem statement, so in particular conditional independence holds for $X_{T}$ and $Y_{T}$ for any specific value of $T=t$. Therefore, by Lemma VI.1 we can find a $\Gamma_{X, Y, U}$ such that

$$
\begin{aligned}
|\mathcal{U}| & \leq|\mathcal{X}||\mathcal{Y}|+1, \\
\Gamma_{X, Y} & =P_{X_{T}, Y_{T}}, \\
I_{\Gamma}(X ; U) & =I_{P}\left(X_{T} ; J, K, T\right) \\
I_{\Gamma}(X, Y ; U) & =I_{P}\left(X_{T}, Y_{T} ; J, K, T\right) .
\end{aligned}
$$

We see from (85) and (86) that $\Gamma_{X, Y, U}$ satisfies the inequalities in (80). What remains is to verify that $\Gamma_{X, Y, U} \in \mathcal{D}$. This is indeed confirmed by applying Lemma VI.2.

$$
\begin{aligned}
\left\|\Gamma_{X, Y}-Q_{X} Q_{Y \mid X}\right\|_{T V} & =\left\|P_{X_{T}, Y_{T}}-Q_{X} Q_{Y \mid X}\right\|_{T V} \\
& \leq\left\|P_{X^{n}, Y^{n}}-\prod Q_{X} Q_{Y \mid X}\right\|_{T V} \\
& <\epsilon .
\end{aligned}
$$

\section{Continuity of $\mathcal{S}_{\epsilon}$ at Zero}

The final step in the proof is to show that the intersection of all $\mathcal{S}_{\epsilon}$ with $\epsilon>0$ is equal to $\mathcal{S}$, a closed set. This may seem like unnecessary detail. It may seem obvious because of how $\mathcal{S}_{\epsilon}$ was deliberately designed, namely $\mathcal{S}_{0}=\mathcal{S}$, and the non-strict inequalities in the definition of $\mathcal{S}$ seem to make it a closed set.

There are a few subtle points to consider. Yes, $\mathcal{S}$ is closed, but this assertion relies on the cardinality bound of $\mathcal{U}$. Also, notice that $\mathcal{S}_{\epsilon}$ allows not only a relaxation in the sum rate but also a relaxation in the set of distributions $\mathcal{D}_{\epsilon}$. We must show that a distribution near the desired input-output distribution does not have a significantly larger achievable rate region, as bounded by $\mathcal{S}_{\epsilon}$. Notice that in other work, such as [2], this complication is avoided by defining the achievable region as the closure of the set of achievable rates and distributions. In the present work, we define the achievable region as the closure of the set of rates for a given distribution-a more precise characterization of the achievable set-which requires this additional precision in the proof.

Lemma VI.5 (Continuity of $\mathcal{S}_{\epsilon}$ at Zero). The epsilon rate regions $\mathcal{S}_{\epsilon}$ decrease to the closed set $\mathcal{S}$ as $\epsilon$ decreases to zero:

$$
\bigcap_{\epsilon>0} \mathcal{S}_{\epsilon}=\mathcal{S} .
$$

Proof: One direction of equality is trivial because $\mathcal{S}_{\epsilon}$ shrinks as $\epsilon$ shrinks and $\mathcal{S}_{0}=\mathcal{S}$ :

$$
\bigcap_{\epsilon>0} \mathcal{S}_{\epsilon} \supset \mathcal{S} .
$$

Notice that $\lim _{\epsilon \rightarrow 0} g(\epsilon)=0$.

First we take care of the easy part. Define $\mathcal{S}_{\epsilon}^{\prime}$ to remove the relaxation in the sum rate:

$$
\mathcal{S}_{\epsilon}^{\prime} \triangleq\left\{\begin{aligned}
\left(R, R_{0}\right) \in \mathcal{R}^{2} & : \exists P_{X, Y, U} \in \mathcal{D}_{\epsilon} \text { s.t. } \\
R & \geq I(X ; U), \\
R_{0}+R & \geq I(X, Y ; U) .
\end{aligned}\right\},
$$

using the same definition for $\mathcal{D}_{\epsilon}$ as in (81). Notice that

$$
\bigcap_{\epsilon>0} \mathcal{S}_{\epsilon} \subset \operatorname{Closure}\left(\bigcap_{\epsilon>0} \mathcal{S}_{\epsilon}^{\prime}\right) \text {. }
$$

This can be verified by contradiction. Suppose $(a, b)$ is in the left-hand side but not the right-hand side. Find the smallest $b^{*}$ such that $\left(a, b^{*}\right)$ is in the right-hand side. Then $b^{*}>b$. Choose $\epsilon$ small enough to exclude $\left(a,\left(b^{*}+b\right) / 2\right)$ from $\mathcal{S}_{\epsilon}^{\prime}$ and so that $g(\epsilon)<\left(b^{*}-b\right) / 2$. Thus, a contradiction.

Now define the function $f: \Delta^{|\mathcal{X}||\mathcal{Y}||\mathcal{U}|-1} \rightarrow \mathcal{R}^{2}$ as follows:

$$
f\left(P_{X, Y, U}\right)=(I(X ; U), I(X, Y ; U)) .
$$

The images $f(D)$ and $f\left(\mathcal{D}_{\epsilon}\right)$ characterize the rate regions $\mathcal{S}$ and $\mathcal{S}_{\epsilon}^{\prime}$. That is, the Pareto optimal points in the images and the respective rate regions are the same. Had the rate regions $\mathcal{S}$ and $\mathcal{S}_{\epsilon}^{\prime}$ been defined with equality for the rate constraints rather than inequality, then they would precisely equal the images $f(D)$ and $f\left(\mathcal{D}_{\epsilon}\right)$.

Notice that

$$
\bigcap_{\epsilon>0} f\left(\mathcal{D}_{\epsilon}\right)=f(D)
$$

because $\bigcap_{\epsilon>0} \mathcal{D}_{\epsilon}=\mathcal{D}$, the sets $\mathcal{D}_{\epsilon}$ are decreasing subsets (as $\epsilon$ decreases) of the compact probability simplex (due to the cardinality bound), and $f$ is a continuous function. This implies,

$$
\bigcap_{\epsilon>0} \mathcal{S}_{\epsilon}^{\prime}=\mathcal{S} .
$$

Finally, $\mathcal{S}$ is closed due to $f$ continuous and $\mathcal{D}$ compact. 


\section{SOFt COVERING GENERALIZATION AND ANALysis}

In this section we present a variety of distribution matching results built from the soft covering principle, provide a simple proof, and investigate error exponents for memoryless sources and channels. We begin with a broad theorem for a general source and channel, from which a subtle improvement to Hayashi's result [42, Lemma 2] is derived. We then illustrate a variety of implications of the theorem.

All statements in this section apply to general distributions, with Radon-Nikodym derivatives substituted where appropriate, although probability mass functions are used for notational simplicity.

\section{A. Soft Covering - General Source and Channel}

The setting of soft covering for a general source and channel is illustrated in Fig. 15. In order to state the theorem, we first define information density and self-information.

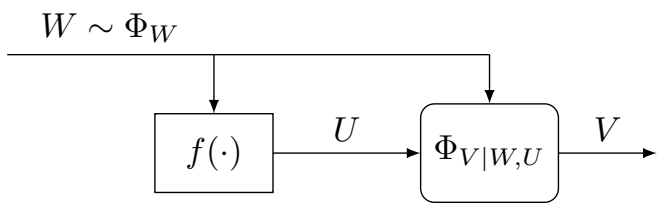

Fig. 15: Theorem VII.1 is a statement about the soft covering principle for a general source and channel. Here a random variable $W$ is the input to a deterministic encoder which produces an output $U$. A channel then acts on the pair $(W, U)$. Given any output distribution consistent with the source and channel, Theorem VII.1 bounds the expected total variation between the desired output distribution and the distribution induced by a randomly constructed encoder.

Definition 6. The information density $i_{\Phi_{U, V}}$ for a joint distribution $\Phi_{U, V}$ is a function on the space $\mathcal{U} \times \mathcal{V}$ specified by the log-likelihood ratio of the joint distribution to the product distribution:

$$
i_{\Phi_{U, V}}(u ; v) \triangleq \log \frac{\Phi_{U, V}(u, v)}{\Phi_{U}(u) \Phi_{V}(v)} .
$$

Definition 7. Self-information is $i(W)=i(W ; W)$.

Notice that the expected value of information density is mutual information and the expected value of self-information is entropy.

$$
\begin{aligned}
\mathbf{E}_{\Phi} i_{\Phi_{U, V}}(U ; V) & =I_{\Phi}(U ; V), \\
\mathbf{E}_{\Phi} i_{\Phi_{W}}(W) & =H_{\Phi}(W) .
\end{aligned}
$$

Theorem VII.1 (Soft covering - General source and channel). For any source distribution $\Phi_{W}$, codebook distribution $\Phi_{U \mid W}$, and channel $\Phi_{V \mid W, U}$, we bound the expected total variation error of the distribution of $V$ induced by a randomly constructed codebook. Let $\mathcal{B}$ be a randomly generated collection of channel inputs $u(w) \in \mathcal{U}, w \in \mathcal{W}$, each drawn independently from $\Phi_{U \mid W}$. Let $P_{V}$ be the output distribution induced by applying the codebook, and let $Q_{V}=\Phi_{V}$ be the desired output distribution $\sum_{w, u} \Phi_{W} \Phi_{U \mid W} \Phi_{V \mid W, U}$. For any $\tau$,

$$
\mathbf{E}\left\|P_{V}-Q_{V}\right\|_{T V} \leq \mathbf{P}_{\Phi}\left(\mathcal{A}_{\tau}^{c}\right)+\delta_{\Phi}(\tau)
$$

where $\mathcal{A}_{\tau}^{c}$ is the complement of $\mathcal{A}_{\tau}$, expectation is with respect to the random codebook, and

$$
\begin{aligned}
\mathcal{A}_{\tau} & \triangleq\left\{(w, u, v): i_{\Phi}(w, u ; v)-i_{\Phi}(w) \leq \tau\right\} \\
\delta_{\Phi}(\tau) & \triangleq \frac{1}{2} \mathbf{E}_{\Phi_{V}} \sqrt{\mathbf{E}_{\Phi_{W, U \mid V}} 2^{i_{\Phi}(W, U ; V)-i_{\Phi}(W)} \mathbf{1}_{\mathcal{A}_{\tau}}} \\
& \leq \frac{1}{2} 2^{\tau / 2} .
\end{aligned}
$$

A simple proof of Theorem VII.1 is given in \$VII-C The significance of (104) over the simpler relaxation (105) is motivated by Hayashi's derivation in [42] of tighter error exponents in the memoryless channel case based on a bound related to (104).

Notice that the setting of Theorem VII.1 is equally general even if the channel $\Phi_{V \mid W, U}$ does not explicitly depend on $W$. The random variable $U$ can be chosen to contain $W$ if necessary, producing the same effect. We choose this presentation because it emphasizes the versatility.

From this theorem we derive a corollary related to known results in the literature. The setting involves the case where $W$ is independent of $U$ and $V$ and uniformly distributed (represented as $J$ in Fig. 16).

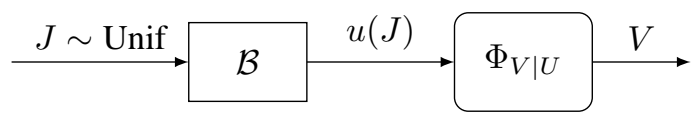

Fig. 16: Soft covering - General channel: Corollary VII.2 arises as a special case of Theorem VII.1 Here, an input to a channel is selected uniformly at random from a codebook $\mathcal{B}$ in order to induce a desired output distribution.

Corollary VII.2 (Soft covering - General channel (see Lemma 2 of [42])). For any channel $\Phi_{V \mid U}$ and codebook distribution $\Phi_{U}$, we bound the expected total variation error of the distribution of $V$ induced by a randomly constructed codebook. Let $\mathcal{B}$ be a randomly generated collection of channel inputs $u(j) \in \mathcal{U}, j=1, \ldots, M$, each drawn independently from $\Phi_{U}$. Let $P_{V}$ be the output distribution induced by selecting a channel input uniformly at random from the codebook, and let $Q_{V}=\Phi_{V}$ be the desired output distribution $\sum_{u \in \mathcal{U}} \Phi_{U} \Phi_{V \mid U}$. For any $\tau$,

$$
\mathbf{E}\left\|P_{V}-Q_{V}\right\|_{T V} \leq \mathbf{P}_{\Phi}\left(\mathcal{A}_{\tau}^{\prime c}\right)+\delta_{\Phi}^{\prime}(\tau),
$$

where $\mathcal{A}_{\tau}^{\prime c}$ is the complement of $\mathcal{A}_{\tau}^{\prime}$, expectation is with respect to the random codebook, and

$$
\begin{aligned}
\mathcal{A}_{\tau}^{\prime} & \triangleq\left\{(u, v): i_{\Phi}(u ; v) \leq \tau\right\} \\
\delta_{\Phi}^{\prime}(\tau) & \triangleq \frac{1}{2 \sqrt{M}} \mathbf{E}_{\Phi_{V}} \sqrt{\mathbf{E}_{\Phi_{U \mid V}} 2^{i_{\Phi}(U ; V)} \mathbf{1}_{\mathcal{A}_{\tau}^{\prime}}} \\
& \leq \frac{1}{2} \sqrt{\frac{2^{\tau}}{M}} .
\end{aligned}
$$

The above corollary differs from Lemma 2 of [42] only in (108), which can be relaxed using Jensen's inequality to arrive at the same statement as in [42], by moving the expectation inside the square root.

A comparison of this bound to the bounds in [17] is given in the appendix. 
For the next two corollaries we consider an arbitrary sequence of channels and use Theorem VII.1 to state sufficient conditions for a random codebook to render an output distribution with arbitrarily high fidelity in the limit. The second of these corollaries specializes to the case of an independent and uniformly distributed source, to recover [17, Theorem 4].

Definition 8. The limit superior in probability with respect to $\Phi$ is defined as

$$
\limsup _{\Phi, n \rightarrow \infty} W_{n} \triangleq \inf \left\{\tau: \mathbf{P}_{\Phi}\left(W_{n}>\tau\right) \rightarrow 0\right\} .
$$

Definition 9. The sup-information rate $\bar{I}_{\Phi}(U ; V)$ for a sequence of joint distributions $\Phi_{U^{(n)}, V^{(n)}}$ of pairs of random variables $\left(U^{(n)}, V^{(n)}\right)$ is defined as

$$
\bar{I}_{\Phi}(U ; V) \triangleq \limsup _{\Phi, n \rightarrow \infty} \frac{1}{n} i_{\Phi_{U^{(n)} ; V^{(n)}}}\left(U^{(n)} ; V^{(n)}\right) .
$$

Corollary VII.3 (Soft covering - Sequence of sources and channels). Given a sequence of sources, channels, and codebook distributions, specified by $\Phi_{W^{(n)}}, \Phi_{V^{(n)} \mid W^{(n)}, U^{(n)}}$, and $\Phi_{U^{(n)} \mid W^{(n)}}$, respectively, for $n=1,2, \ldots$, let $\mathcal{B}^{(n)}$ be a randomly generated collection of channel inputs $u^{(n)}\left(w^{(n)}\right) \in$ $\mathcal{U}^{(n)} \forall w^{(n)} \in \mathcal{W}^{(n)}$, each drawn independently from $\Phi_{U^{(n)} \mid W^{(n)}}$. Let $P_{V^{(n)}}$ be the output distribution induced by applying the codebook, and let $Q_{V^{(n)}}=\Phi_{V^{(n)}}$ be the desired output distribution $\sum_{u^{(n)} \in \mathcal{U}^{(n)}} \Phi_{U^{(n)}} \Phi_{V^{(n)} \mid U^{(n)}}$. The distribution $P_{V^{(n)}}$ is random because the codebook $\mathcal{B}^{(n)}$ is random.

Then,

$$
\begin{gathered}
\lim _{\Phi, n \rightarrow \infty} i_{\Phi}\left(W^{(n)}, U^{(n)} ; V^{(n)}\right)-i_{\Phi}\left(W^{(n)}\right)=-\infty \\
\Downarrow \\
\lim _{n \rightarrow \infty} \mathbf{E}\left\|P_{V^{(n)}}-Q_{V^{(n)}}\right\|_{T V}=0 .
\end{gathered}
$$

Corollary VII.4 (Soft covering - Sequence of channels [17, Theorem 4]). Given a sequence of channels and codebook distributions, specified by $\Phi_{V^{(n)} \mid U^{(n)}}$ and $\Phi_{U^{(n)}}$ for $n=$ $1,2, \ldots$, let $\mathcal{B}^{(n)}$ be a randomly generated collection of $2^{n R}$ channel inputs in $\mathcal{U}^{(n)}$, each drawn independently from $\Phi_{U^{(n)}}$. Let $P_{V^{(n)}}$ be the output distribution induced by selecting a channel input uniformly at random from the codebook, and let $Q_{V^{(n)}}=\Phi_{V^{(n)}}$ be the desired output distribution $\sum_{u^{(n)} \in \mathcal{U}^{(n)}} \Phi_{U^{(n)}} \Phi_{V^{(n)} \mid U^{(n)}}$. The distribution $P_{V^{(n)}}$ is random because the codebook $\mathcal{B}^{(n)}$ is random.

Then,

$$
\begin{gathered}
R>\bar{I}_{\Phi}(U ; V) \\
\Downarrow \\
\lim _{n \rightarrow \infty} \mathbf{E}\left\|P_{V^{(n)}}-Q_{V^{(n)}}\right\|_{T V}=0 .
\end{gathered}
$$

\section{B. Implications of Soft Covering}

From Theorem VII.1 we can derive a variety of results about randomly generated but deterministic encoders used to synthesizing a stochastic process. In this section we highlight some examples involving memoryless channels. For convenience, we will assume that all random variables are discrete. However, only corollaries VII.6 and VII.8 require any modification for general distributions.

Through simple entropy arguments, most of the required rates in the corollaries of this section can be shown to be tight, up to a null space in the channel transition matrix, as outlined in the last section of the appendix.

First, consider as a starting point an i.i.d. sequence $W^{n}$ and a memoryless channel $\Phi_{V, U \mid W}$ depicted in Fig. 17. A deterministic but randomly generated encoder receives both the source $W^{n}$ and a uniformly distributed variable $J \in\left[2^{n R}\right]$. The following corollary, which serves as a conceptual building block for the remainder of this section, states sufficient rates for the channel output $V^{n}$ to be i.i.d. in the limit of large $n$. Notice that the corollary states that the entropy of the source $W$ directly replaced some (or all) of the required random bits $J$ fed into the deterministic encoder.

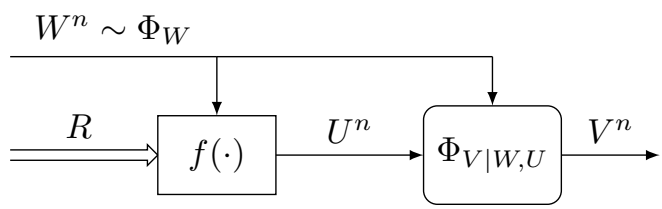

Fig. 17: (Corollary VII.5): A deterministic encoder, represented by $f(\cdot)$, is randomly generated according to $\Phi_{U \mid W}$. If $R>I(W, U ; V)-H(W)$ then the output $V^{n}$ is i.i.d. in the limit of large $n$.

Corollary VII.5. Consider any i.i.d. source distribution specified by $\Phi_{W}$, codebook distribution $\Phi_{U \mid W}$, and memoryless channel $\Phi_{V \mid W, U}$. Let $\mathcal{B}^{(n)}$ be a randomly generated collection of channel inputs $u^{n}\left(w^{n}, j\right) \in \mathcal{U}^{n}$, for all $w^{n} \in \mathcal{W}^{n}$ and $j \in$ $\left[2^{n R}\right]$, each drawn independently from $\prod \Phi_{U \mid W}$. Let $P_{V^{n}}$ be the output distribution induced by applying the codebook in the configuration in Fig. 17 and let $Q_{V^{n}}=\Phi_{V^{n}}$ be the desired i.i.d. output distribution specified by $\sum_{w, u} \Phi_{W} \Phi_{U \mid W} \Phi_{V \mid W, U}$.

Then,

$$
\begin{gathered}
R>I_{\Phi}(W, U ; V)-H_{\Phi}(W) \\
\Downarrow \\
\lim _{n \rightarrow \infty} \mathbf{E}\left\|P_{V^{n}}-Q_{V^{n}}\right\|_{T V}=0,
\end{gathered}
$$

and convergence occurs exponentially quickly in $n$.

The above Corollary VII.5 is an immediate consequence of Corollary VII.3 and the law of large numbers, where $W^{n}$ and the uniformly distributed index $J$ are together defined as the source in Corollary VII.3, and $J$ is independent of the codebook distribution and the channel. Exponential convergence follows from the technique of $\$$ VII-D as with the remaining corollaries of this section.

Next, consider locally synthesizing a memoryless channel by making use of a random index and another memoryless channel as the stochastic resources. The setting is depicted in Fig. 18. The case where the channel output is equal to the codebook output has been studied in the literature (e.g. [35]).

For the statement of the corollary, we first define empirical distribution. 


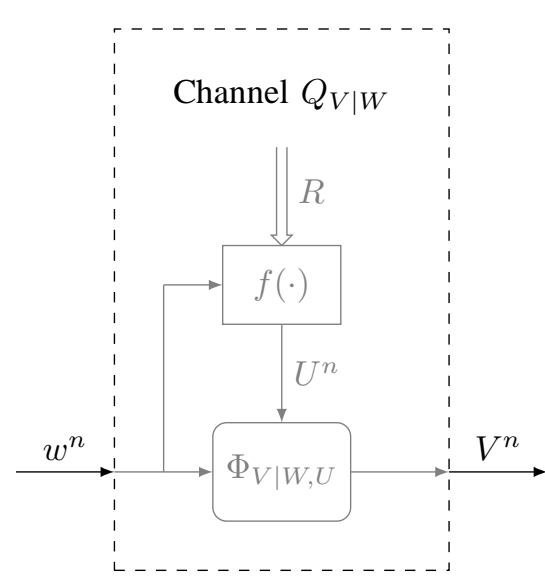

Fig. 18: Local Channel Synthesis (Corollary VII.6): A memoryless channel $Q_{V \mid W}$ is locally synthesized with $I(U ; V \mid W)$ bits of randomness and a channel $\Phi_{V \mid W, U}$.

Definition 10. The empirical distribution of a sequence $w^{n} \in$ $\mathcal{W}^{n}$ is a probability mass function expressing the frequencies of each $w \in \mathcal{W}$, denoted

$$
\mathbb{P}_{w^{n}}(w) \triangleq \frac{1}{n} \sum_{t=1}^{n} \mathbf{1}\left(w_{t}=w\right) .
$$

Corollary VII.6 (Local channel synthesis). Consider a codebook distribution $\Phi_{U \mid W}$ and memoryless channel $\Phi_{V \mid W, U}$. Let $\mathcal{B}^{(n)}$ be a randomly generated collection of channel inputs $u^{n}\left(w^{n}, j\right) \in \mathcal{U}^{n}$, for all $w^{n} \in \mathcal{W}^{n}$ and $j \in\left[2^{n R}\right]$, each drawn independently from $\prod \Phi_{U \mid W}$. Let $P_{V^{n} \mid W^{n}}$ be the conditional distribution induced by applying the codebook in the configuration in Fig. 18] and let $Q_{V^{n} \mid W^{n}}=\Phi_{V^{n} \mid W^{n}}$ be the desired memoryless conditional distribution specified by $\Phi_{V \mid W}=\sum_{u} \Phi_{U \mid W} \Phi_{V \mid W, U}$.

For all $w^{n}$ having empirical distribution $\mathbb{P}_{w^{n}}$ such that $R>$ $I_{\mathbb{P} \Phi}(U ; V \mid W)+\gamma_{n}$, where mutual information is calculated with respect to $\mathbb{P}_{w^{n}} \Phi_{U, V \mid W}$, and $\gamma_{n} \in \omega(1 / \sqrt{n})$, the expected value of the total variation between the induced conditional distribution and the desired conditional distribution vanishes uniformly as $n$ grows. That is, there exists $\epsilon_{n}$ going to zero, depending only on $\Phi_{U, V \mid W}$ and $\gamma_{n}$, such that

$$
\begin{gathered}
R>I_{\mathbb{P} \Phi}(U ; V \mid W)+\gamma_{n} \\
\Downarrow \\
\mathbf{E}\left\|P_{V^{n} \mid W^{n}=w^{n}}-Q_{V^{n} \mid W^{n}=w^{n}}\right\|_{T V}<\epsilon_{n} \rightarrow 0 .
\end{gathered}
$$

Furthermore, if $\gamma_{n}$ is a constant $\gamma>0$, then $\epsilon_{n}$ can be chosen to go to zero exponentially fast.

Notice that if we had defined $W^{n}$ to be an i.i.d. source and asked that the induced joint distribution $P_{W^{n}, V^{n}}$ approach the desired i.i.d. distribution $Q_{W^{n}, V^{n}}$, the result in Corollary VII.6 would be a special case of Corollary VII.5. This occurs by defining the channel in Corollary VII.5 to be $\Phi_{W, V \mid U, W}$, which outputs $W$ as well as $V$. However, Corollary VII.6 is stronger in that it states that the conditional distribution of $V^{n}$ given $w^{n}$ will be accurate for all $w^{n}$ with the appropriate empirical distribution, rather than on average over $w^{n}$.
Proof of Corollary VII.6 follows from Corollary VII.4 Define the distribution $\prod_{t=1}^{n} \Phi_{U \mid W=w_{t}}$ as the codebook distribution in Corollary VII.4 and the conditional distribution $\prod_{t=1}^{n} \Phi_{V \mid U, W=w_{t}}$ as the channel. The statement of Corollary VII.6 follows from Chebyshev's inequality, since the input-output information density has mean $n I_{\mathbb{P} \Phi}(U ; V \mid W)$ and standard deviation $O(\sqrt{n})$.

Next, we make a simple extension to LemmaIV.1 that incorporates memoryless sources other than a uniformly distributed random index. Fig. 19 depicts a deterministic encoder that has access to both a random index at rate $R$ and an i.i.d. source $W^{r n}$ at potentially a different rate than the output, specified by $r$. The case where the channel $\Phi_{V \mid U}$ is the identity channel has been studied in depth in the literature (see [52, Chapter 2]).

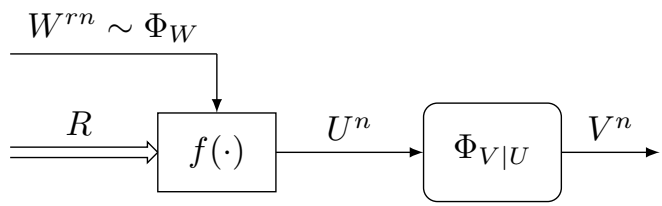

Fig. 19: (Corollary VII.7): A deterministic encoder, represented by $f(\cdot)$, is randomly generated according to $\Phi_{U}$. If $R+r H(W)>I(U ; V)$ then the output $V^{n}$ is i.i.d. in the limit of large $n$.

Corollary VII.7. Consider any i.i.d. source distribution specified by $\Phi_{W}$, codebook distribution $\Phi_{U}$, and memoryless channel $\Phi_{V \mid U}$. Let $\mathcal{B}^{(n)}$ be a randomly generated collection of channel inputs $u^{n}\left(w^{r n}, j\right) \in \mathcal{U}^{n}$, for all $w^{r n} \in \mathcal{W}^{r n}$ and $j \in\left[2^{n R}\right]$, each drawn independently from $\prod \Phi_{U}$. Let $P_{V^{n}}$ be the output distribution induced by applying the codebook in the configuration in Fig. 19] and let $Q_{V^{n}}=\Phi_{V^{n}}$ be the desired i.i.d. output distribution specified by $\sum_{u} \Phi_{U} \Phi_{V \mid U}$.

Then

$$
\begin{gathered}
R+r H_{\Phi}(W)>I_{\Phi}(U ; V) \\
\Downarrow \\
\lim _{n \rightarrow \infty} \mathbf{E}\left\|P_{V^{n}}-Q_{V^{n}}\right\|_{T V}=0,
\end{gathered}
$$

and convergence occurs exponentially quickly in $n$.

The above Corollary VII.7 is an immediate consequence of Corollary VII.3 and the law of large numbers, where $W^{r n}$ and the uniformly distributed index $J$ are together defined as the source in Corollary VII.3, both independent of the codebook distribution and the channel. Notice that in the synchronous case where $r=1$ there is actually flexibility in designing the codebook. The result still holds if the codebook is constructed from any conditional distribution $\Phi_{U \mid W}$ resulting in the same marginal distribution on $U$.

In the final derivation of this section, we consider two sources of random bits feeding into two separate deterministic encoders. The output of the first encoder is fed into the second encoder, as in Fig. 20. The codebooks together form a superposition codebook. A similar superposition construction was analyzed in [18]. 


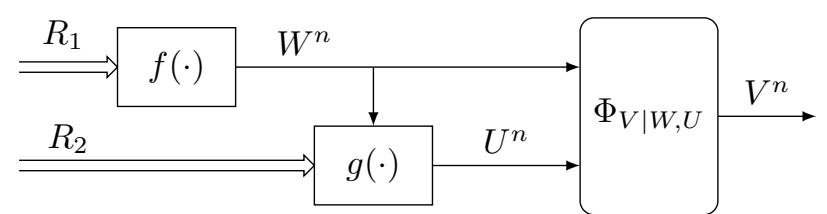

Fig. 20: Superposition Encoding: Two deterministic encoders, represented by $f(\cdot)$ and $g(\cdot)$, are randomly generated according to $\Phi_{W}$ and $\Phi_{U \mid W}$. Sufficient rates for producing an i.i.d. output sequence $V^{n}$ in the limit of large $n$ are given in Corollary VII.8.

Corollary VII.8 (Superposition). Consider two codebook distributions $\Phi_{W}$ and $\Phi_{U \mid W}$ and a memoryless channel $\Phi_{V \mid W, U}$. Let $\mathcal{B}_{1}^{(n)}$ be a randomly generated collection of sequences $w^{n}(j) \in \mathcal{W}^{n}$, for all $j \in\left[2^{n R_{1}}\right]$, each drawn independently from $\prod \Phi_{W}$, and let $\mathcal{B}_{2}^{(n)}$ be a randomly generated collection of sequences $u^{n}\left(w^{n}, k\right) \in \mathcal{U}^{n}$, for all $w^{n} \in \mathcal{B}_{1}^{(n)}$ and $k \in\left[2^{n R_{2}}\right]$, each drawn independently from $\prod \Phi_{U \mid W}$. Let $P_{V^{n}}$ be the output distribution induced by applying the codebooks in the configuration of Fig. 20. and let $Q_{V^{n}}=$ $\Phi_{V^{n}}$ be the desired i.i.d. output distribution specified by $\sum_{w, u} \Phi_{W} \Phi_{U \mid W} \Phi_{V \mid W, U}$

Then,

$$
\begin{aligned}
& R_{1}>I(W ; V), \\
& R_{2}>I(W, U ; V)-H(W), \\
& R_{1}+R_{2}>I(W, U ; V) \\
& \Downarrow \\
& \lim _{n \rightarrow \infty} \mathbf{E}\left\|P_{V^{n}}-Q_{V^{n}}\right\|_{T V}=0,
\end{aligned}
$$

and convergence occurs exponentially quickly in $n$.

To prove Corollary VII.8, we need only show how to achieve the two corner points. First consider rates exceeding $\left(R_{1}, R_{2}\right)=(I(W ; V), I(U ; V \mid W))$. By Corollary VII.6, the second encoder $g(\cdot)$, operating at rate $R_{2}>I(U ; V \mid W)$, synthesizes a memoryless channel from $W$ to $V$, universally for all $w^{n}$ with the appropriate empirical distribution, which will occur with exponentially high probability in the random codebook $\mathcal{B}_{1}^{(n)}$. Thus, the first encoder need only operate at a rate $R_{1}>I(W ; V)$ to induce an i.i.d. output, according to Lemma IV.1

Next consider rates exceeding $\left(R_{1}, R_{2}\right)=$ $(H(W), I(W, U ; V)-H(W))$. According to Corollary VII.7 with the identity channel, the first encoder $f(\cdot)$, operating at rate $R_{1}>H(W)$, renders the sequence $W^{n}$ i.i.d. in the limit of large $n$. With $W^{n}$ an i.i.d. sequence, Corollary VII.5 declares the rate $R_{2}>I(W, U ; V)-H(W)$ to be sufficient to produce an i.i.d. output.

The proof is completed by drawing on lemmas $\mathrm{V} .1$ and $\mathrm{V} .2$ and noting that the expected total variation decays exponentially quickly in each of the supporting lemmas and corollaries.

Notice the similarity between Corollary VII.8 and the "generalization of Lemma 6.1 of [1]" in [18]. The difference is the constraint on $R_{2}$. In [18], the index $J$ is given to second encoder, instead of only the sequence $w^{n}(J)$. Notice that when
$R_{1}<H(W)$ the index $J$ will be uniquely determined from $w^{n}(J)$ with high probability. Thus, the required rate region only differs when $R_{1} \geq H(W)$, after which increases to $R_{1}$ have no further effect in the situation of Corollary VII.8

\section{Proof of Theorem VII.1}

1) Explanation: This proof of soft covering is enabled by two important steps. First is to define a well-behaved "typical set" and separate the induced output distribution into two parts accordingly. For this we use $\mathcal{A}_{\tau}$ defined in (103) of the theorem. The typical set is intended to contain the bulk of the probability mass. To analyze the total variation contribution from the typical part, the key step is to use Jensen's inequality in the following way: $\mathbf{E}|\cdot| \leq \sqrt{\mathbf{E}(\cdot)^{2}}$. This allows for the variance analysis in (121).

The steps of this proof are also nearly the same steps used by Hayashi in [42]. The difference is that Hayashi applies Jensen's inequality to the square-root function twice, which can be avoided by changing the order of summation.

In [23, Lemma 19], we use these same steps to directly prove the digital rate case in Lemma [V.1, which is the basic soft covering lemma pertaining to a memoryless channel and uniform and independent source distribution. Due to those simplifying assumptions, some readers may find the proof in [23] easier to internalize because of the reduced notation and more familiar definition of the typical set.

One might also gain intuition by substituting $\Phi_{W}(w)=$ $\frac{1}{M}$ for $w=1, \ldots, M, \Phi_{U \mid W}(u \mid w)=\Phi_{U}(u)$, and $\Phi_{V \mid W, U}(v \mid w, u)=\Phi_{V \mid U}(v \mid u)$ throughout this proof, to directly prove Corollary VII.2, which is the form relevant to channel resolvability.

This proof is stated in terms of discrete random variables, but it can be modified for general random variables with the appropriate use of the Radon-Nikodym derivative. In the general proof, care must be taken in several places, including expressions such as the definition of $\mathcal{A}_{\tau}$ in (103). Comparisons of two infinite values are considered to not satisfy the inequality and thus are not included in $\mathcal{A}_{\tau}$.

2) Proof: Recall that we are given three distributions: the source distribution $\Phi_{W}$, the codebook distribution $\Phi_{U \mid W}$, and the channel $\Phi_{V \mid W, U}$. The source and channel are stochastic according to their prescribed distributions. However, the encoder produces a deterministic output $u(w)$. The induced output distribution is

$$
P_{V}(v)=\sum_{w \in \mathcal{W}} \Phi_{W}(w) \Phi_{V \mid W, U}(v \mid w, u(w)) .
$$

The theorem bounds the total variation between the desired output distribution $Q_{V}=\Phi_{V}$ that would result from a stochastic encoder that operates according to $\Phi_{U \mid W}$ and the output distribution induced by the deterministic encoder in (113). Specifically, we bound the expected total variation when the codebook entries are generated randomly and independently according to the desired conditional distribution $\Phi_{U \mid W}$.

For brevity, we omit most subscripts of distributions in this proof, which are clear in context. Thus, $\Phi(w)$ means $\Phi_{W}(w)$. 
We start by separating out the contribution to $P_{V}$ coming from typical triples $(w, u, v) \in \mathcal{A}_{\tau}$. Let us define two functions on $\mathcal{V}$ that sum to $P_{V}$ :

$$
\begin{aligned}
& P_{1}(v) \triangleq \sum_{w \in \mathcal{W}} \Phi(w) \Phi(v \mid w, U(w)) \mathbf{1}_{\mathcal{A}_{\tau}}(w, U(w), v),(11 \\
& P_{2}(v) \triangleq \sum_{w \in \mathcal{W}} \Phi(w) \Phi(v \mid w, U(w)) \mathbf{1}_{\mathcal{A}_{\tau}^{c}}(w, U(w), v),
\end{aligned}
$$

where 1 represents the indicator function, $\mathcal{A}_{\tau}^{c}$ is the complement of $\mathcal{A}_{\tau}$, and $U(w)$ is the codebook entry for source $w$ and is a capital letter to represent that the codebook is randomly generated. Under these definitions,

$$
P_{V}=P_{1}+P_{2} .
$$

An important observation about the induced output distribution $P_{V}$, which is random because the codebook is random, is that it is unbiased with respect to the desired output distribution $Q_{V}$ :

$$
\begin{aligned}
\mathbf{E} P_{V}(v) & \stackrel{(a)}{=} \sum_{w \in \mathcal{W}} \Phi(w) \mathbf{E} \Phi(v \mid w, U(m)) \\
& \stackrel{(b)}{=} \sum_{w \in \mathcal{W}} \Phi(w) \sum_{u \in \mathcal{U}} \Phi(u \mid w) \Phi(v \mid w, u) \\
& =\Phi(v) \\
& =Q_{V}(v),
\end{aligned}
$$

where (a) is an application of linearity of expectation to the definition of $P_{V}$, and (b) arises by inserting the distribution of the codebook.

We separate the total variation $\mathbf{E}\left\|P_{V}-Q_{V}\right\|_{T V}$ into two parts:

$$
\begin{aligned}
\mathbf{E}\left\|P_{V}-Q_{V}\right\|_{T V} & \\
= & \mathbf{E}\left\|P_{V}-\mathbf{E} P_{V}\right\|_{T V} \\
= & \frac{1}{2} \sum_{v \in \mathcal{V}} \mathbf{E}\left|P_{V}(v)-\mathbf{E} P_{V}(v)\right| \\
\stackrel{(a)}{\leq} & \frac{1}{2} \sum_{v \in \mathcal{V}} \mathbf{E}\left|P_{1}(v)-\mathbf{E} P_{1}(v)\right| \\
& +\frac{1}{2} \sum_{v \in \mathcal{V}} \mathbf{E}\left|P_{2}(v)-\mathbf{E} P_{2}(v)\right|,
\end{aligned}
$$

where (a) is due to the triangle inequality.

The first sum in $(118)$ is the interesting one to consider, so we save it for last. The second sum is easy to handle and is small as long as the typical set $\mathcal{A}_{\tau}$ is likely. Starting by again making use of the triangle inequality,

$$
\begin{aligned}
\frac{1}{2} \sum_{v \in \mathcal{V}} \mathbf{E}\left|P_{2}(v)-\mathbf{E} P_{2}(v)\right| \\
\quad \leq \sum_{v \in \mathcal{V}} \mathbf{E} P_{2}(v) \\
\quad=\sum_{v \in \mathcal{V}} \mathbf{E}\left(\sum_{w \in \mathcal{W}} \Phi(w) \Phi(v \mid w, U(m)) \mathbf{1}_{\mathcal{A}_{\tau}^{c}}(w, U(w), v)\right) \\
\quad=\sum_{(w, v) \in \mathcal{W} \times \mathcal{V}} \Phi(w) \mathbf{E}\left(\Phi(v \mid w, U(m)) \mathbf{1}_{\mathcal{A}_{\tau}^{c}}(w, U(w), v)\right)
\end{aligned}
$$

$$
\begin{aligned}
& =\sum_{(w, v) \in \mathcal{W} \times \mathcal{V}} \Phi(w) \sum_{u \in \mathcal{U}} \Phi(u \mid w) \Phi(v \mid w, u) \mathbf{1}_{\mathcal{A}_{\tau}^{c}}(w, u, v) \\
& =\mathbf{P}_{\Phi}\left(\mathcal{A}_{\tau}^{c}\right)
\end{aligned}
$$

The remaining term in 118 deals with only typical triples. To bound this term we appeal to a variance bound with the help of Jensen's inequality:

$$
\begin{aligned}
\mathbf{E}\left|P_{1}(v)-\mathbf{E} P_{1}(v)\right| & \leq \sqrt{\mathbf{E}\left(P_{1}(v)-\mathbf{E} P_{1}(v)\right)^{2}} \\
& =\sqrt{\operatorname{Var} P_{1}(v)} .
\end{aligned}
$$

Since the codebook is randomly constructed independently for each $w \in \mathcal{W}$, the variance of $P_{1}(v)$ separates as

$$
\begin{aligned}
\operatorname{Var} & P_{1}(v) \\
= & \operatorname{Var}\left(\sum_{w \in \mathcal{W}} \Phi(w) \Phi(v \mid w, U(m)) \mathbf{1}_{\mathcal{A}_{\tau}}(w, U(w), v)\right) \\
\stackrel{(a)}{=} & \sum_{w \in \mathcal{W}} \operatorname{Var}\left(\Phi(w) \Phi(v \mid w, U(w)) \mathbf{1}_{\mathcal{A}_{\tau}}(w, U(w), v)\right) \\
\leq & \sum_{w \in \mathcal{W}} \mathbf{E}\left(\Phi(w) \Phi(v \mid w, U(w)) \mathbf{1}_{\mathcal{A}_{\tau}}(w, U(w), v)\right)^{2} \\
= & \sum_{(w, u) \in \mathcal{W} \times \mathcal{U}} \Phi(u \mid w)\left(\Phi(w) \Phi(v \mid w, u) \mathbf{1}_{\mathcal{A}_{\tau}}(w, u, v)\right)^{2} \\
= & \sum_{(w, u) \in \mathcal{W} \times \mathcal{U}} \Phi(u \mid w) \Phi^{2}(w) \Phi^{2}(v \mid w, u) \mathbf{1}_{\mathcal{A}_{\tau}} \\
= & \sum_{(w, u) \in \mathcal{W} \times \mathcal{U}} \Phi(w, u, v) \Phi(w) \Phi(v \mid w, u) \mathbf{1}_{\mathcal{A}_{\tau}} \\
= & \Phi^{2}(v) \sum_{(w, u) \in \mathcal{W} \times \mathcal{U}} \Phi(w, u \mid v) \Phi(w) \frac{\Phi(v \mid w, u)}{\Phi(v)} \mathbf{1}_{\mathcal{A}_{\tau}} \\
= & \Phi^{2}(v) \mathbf{E}_{\Phi_{W, U \mid V}} 2^{i_{\Phi}(W, U ; V)-i_{\Phi}(W)} \mathbf{1}_{\mathcal{A}_{\tau} .}
\end{aligned}
$$

Equality (a) is due to the independence of the items in the codebook.

The conclusion with respect to the first term in (118) is

$$
\begin{aligned}
& \frac{1}{2} \sum_{v \in \mathcal{V}} \mathbf{E}\left|P_{1}(v)-\mathbf{E} P_{1}\left(v^{n}\right)\right| \\
& \leq \frac{1}{2} \sum_{v \in \mathcal{V}} \sqrt{\Phi^{2}(v) \mathbf{E}_{\Phi_{W, U \mid V}} 2^{i_{\Phi}(W, U ; V)-i_{\Phi}(W)} \mathbf{1}_{\mathcal{A}_{\tau}}} \\
& =\frac{1}{2} \mathbf{E}_{\Phi_{V}} \sqrt{\mathbf{E}_{\Phi_{W, U \mid V}} 2^{i_{\Phi}(W, U ; V)-i_{\Phi}(W)} \mathbf{1}_{\mathcal{A}_{\tau}}} \\
& \stackrel{\text { (a) }}{\leq} \frac{1}{2} \mathbf{E}_{\Phi_{V}} \sqrt{\mathbf{E}_{\Phi_{W, U \mid V}} 2^{\tau}} \\
& =\frac{1}{2} 2^{\tau / 2} \text {, }
\end{aligned}
$$

where (a) results from the definition of $\mathcal{A}_{\tau}$.

\section{Exponents of Total Variation}

In this section we derive bounds on the exponential rate of decay of total variation error for soft covering in the memoryless case. The bounds are stated in terms of discrete distributions but apply generally.

Lemma VII.9 (Exponent for Theorem VII.1). Consider the n-fold memoryless extension of Theorem VII.1 That is, the 
source distribution of Fig. [15 is the i.i.d. distribution according to $\Phi_{W}$, the codebook distribution is the memoryless distribution according to $\Phi_{U \mid W}$, and the channel is memoryless according to $\Phi_{V \mid W, U}$.

If $H_{\Phi}(W)>I_{\Phi}(W, U ; V)$, then the expected total variation error vanishes exponentially fast:

$$
\mathbf{E}\left\|P_{V^{n}}-Q_{V^{n}}\right\|_{T V} \leq \frac{3}{2} \exp (-\gamma n),
$$

where

$$
\begin{aligned}
\gamma \triangleq & \max _{\beta, \beta^{\prime} \geq 0} \frac{-\beta^{\prime}}{2 \beta+\beta^{\prime}} \log \mathbf{E}_{\Phi} Z^{\beta} \\
& +\frac{-\beta}{2 \beta+\beta^{\prime}} \log \left(\mathbf{E}_{\Phi_{V}} \sqrt{\mathbf{E}_{\Phi_{W, U \mid V}} Z^{1-\beta^{\prime}}}\right)^{2},
\end{aligned}
$$

and

$$
Z \triangleq \Phi_{W}(W) \frac{\Phi_{V \mid W, U}(V \mid W, U)}{\Phi_{V}(V)} .
$$

The digital rate case in Lemma IV.1, which is the basic soft covering lemma, is derived by substituting $\Phi_{W}=2^{-R}$, $\Phi_{U \mid W}=\Phi_{U}$, and $\Phi_{V \mid W, U}=\Phi_{V \mid U}$. Technically, $2^{R}$ is restricted to be an integer for this substitution, but this is merely a technicality that is avoided by a direct proof Lemma IV.1 from Corollary VII.2 The result of this substitution provides a new achievable exponent for channel resolvability. We obtain,

$$
\begin{aligned}
\gamma= & \max _{\alpha \geq 1, \alpha^{\prime} \leq 2} \frac{\alpha-1}{2 \alpha-\alpha^{\prime}}\left(R-\breve{I}_{\Phi, \alpha}(U ; V)\right. \\
& \left.+\left(\alpha^{\prime}-1\right)\left(\breve{I}_{\Phi, \alpha}(U ; V)-\bar{I}_{\Phi, \alpha^{\prime}}(U ; V)\right)\right)
\end{aligned}
$$

where $\breve{I}_{\Phi, \alpha}(U ; V)$ is the Rényi divergence of order $\alpha$ between the joint distribution and the product distribution, defined as

$$
\breve{I}_{\Phi, \alpha}(U ; V) \triangleq \frac{1}{\alpha-1} \log \mathbf{E}_{\Phi}\left(\frac{\Phi_{U, V}(U, V)}{\Phi_{U}(U) \Phi_{V}(V)}\right)^{\alpha-1}
$$

and the formula for $\bar{I}_{\Phi, \alpha^{\prime}}(U ; V)$ is

$$
\frac{1}{\alpha^{\prime}-1} \log \left(\mathbf{E}_{\Phi_{V}} \sqrt{\mathbf{E}_{\Phi_{U \mid V}}\left(\frac{\Phi_{U, V}(U, V)}{\Phi_{U}(U) \Phi_{V}(V)}\right)^{\alpha^{\prime}-1}}\right)^{2} .
$$

Notice that the quantity $\bar{I}_{\Phi, \alpha^{\prime}}(U ; V)$ defined above is smaller than $\breve{I}_{\Phi, \alpha^{\prime}}(U ; V)$ and reminiscent of quantities used by Gallager, Arikan, and Arimoto, compared by Csiszár in [53].

Some weaker exponents are also of interest. Define

$$
\begin{aligned}
& \hat{\gamma} \triangleq \max _{\alpha \in[1,2]} \frac{-1}{\alpha} \log \mathbf{E}_{\Phi} Z^{\alpha-1}, \\
& \hat{\gamma} \triangleq \max _{\alpha \geq 1} \frac{-1}{\alpha+(\alpha-1)} \log \mathbf{E}_{\Phi} Z^{\alpha-1} .
\end{aligned}
$$

One can verify that these exponents are positive (implying exponential decay) if the condition $H_{\Phi}(W)>I_{\Phi}(W, U ; V)$ is satisfied by evaluating the derivative of $\mathbf{E}_{\Phi} Z^{\alpha-1}$ at $\alpha=1$.

We arrive at $\hat{\gamma}$ by first relaxing the second term of (124), using Jensen's inequality to move the expected value inside the square-root. We then make the assignment $\alpha-1=\beta=1-\beta^{\prime}$. This assignment can be viewed as an additional relaxation of the bound, although it appears numerically to be optimal and analytically to be at least locally optimal.

When specialized to the digital rate case in Lemma IV.1 the exponent $\hat{\gamma}$ recovers Hayashi's result in [42]. That is,

$$
\hat{\gamma}=\max _{\alpha \in[1,2]} \frac{\alpha-1}{\alpha}\left(R-\breve{I}_{\Phi, \alpha}(U ; V)\right) .
$$

Recall that $\breve{I}_{\Phi, \alpha}(U ; V)$ is defined in (127).

On the other hand, choosing $\beta^{\prime}=1$ eliminates the second term of (124), yielding $\hat{\hat{\gamma}}$ as a suboptimal choice. This exponent corresponds to the relaxed bound in (105), as is mentioned in the proof below. Thus, under that simple relaxation, the best exponential bound that can be attained, when specialized to the digital rate case in Lemma IV.1, is

$$
\hat{\hat{\gamma}}=\max _{\alpha \geq 1} \frac{\alpha-1}{\alpha+(\alpha-1)}\left(R-\breve{I}_{\Phi, \alpha}(U ; V)\right) .
$$

Using the Taylor expansion, it can be shown that $\gamma, \hat{\gamma}$, and $\hat{\gamma}$ of (126), (131), and (132) are approximately equal when $R-I_{\Phi}(U ; V)$ is small. For example, define $\Delta_{I}$ to be the first derivative of $\breve{I}_{\Phi, \alpha}(U ; V)$ with respect to $\alpha$ at $\alpha=1$. Assuming $\Delta_{I} \neq 0$,

$$
\gamma \approx \hat{\gamma} \approx \hat{\hat{\gamma}} \approx \frac{1}{2} \Delta_{I}\left(R-I_{\Phi}(U ; V)\right)^{2} .
$$

However, when $R-I_{\Phi}(U ; V)$ is large, the optimizing choices of $\alpha$ and $\alpha^{\prime}$ go to extreme values, and

$$
\begin{aligned}
& \gamma \approx \frac{1}{2}\left(R-\bar{I}_{\Phi, 2}(U ; V)\right), \\
& \hat{\gamma} \approx \frac{1}{2}\left(R-\breve{I}_{\Phi, 2}(U ; V)\right), \\
& \hat{\hat{\gamma}} \approx \frac{1}{2}\left(R-\breve{I}_{\Phi, \infty}(U ; V)\right),
\end{aligned}
$$

where $\bar{I}_{\Phi, \alpha}(U ; V)$ is defined in (128).

Proof of Lemma VII.9. Let $\tau$ in the bound of Theorem VII.1 grow linearly with $n$. That is,

$$
\tau=n \tau^{\prime}
$$

This proof follows the technique of Hayashi in [42]. Beginning with the first term of the bound of Theorem VII.1, for all $\beta \geq 0$,

$$
\begin{aligned}
& \mathbf{P}_{\Phi}\left(\mathcal{A}_{\tau}^{c}\right) \\
& \quad=\mathbf{P}_{\Phi}\left(i_{\Phi}\left(W^{n}, U^{n} ; V^{n}\right)-i_{\Phi}\left(W^{n}\right)>n \tau^{\prime}\right) \\
& \stackrel{(a)}{=} \mathbf{P}_{\Phi}\left(\frac{1}{n} \sum_{t=1}^{n}\left(i_{\Phi}\left(W_{t}, U_{t} ; V_{t}\right)-i_{\Phi}\left(W_{t}\right)\right)>\tau^{\prime}\right) \\
& \stackrel{(b)}{\leq} \exp \left(n\left(\log \mathbf{E}_{\Phi} 2^{\beta\left(i_{\Phi}(W, U ; V)-i_{\Phi}(W)\right)-\beta \tau^{\prime}}\right)\right) \\
& =\exp \left(n\left(\log \mathbf{E}_{\Phi} Z^{\beta}-\beta \tau^{\prime} \log 2\right)\right),
\end{aligned}
$$

where (a) is due to the i.i.d. property of the distribution and (b) is the Chernoff bound, which is tight to first order in the exponent.

Next consider the following upper bound on the indicator function:

$$
\mathbf{1}_{\{a \leq b\}}(a, b) \leq\left(\frac{b}{a}\right)^{\beta^{\prime}} \quad \forall a, b, \beta^{\prime} \geq 0 .
$$


By applying

$$
\begin{aligned}
a & =2^{i_{\Phi}\left(w^{n}, u^{n} ; v^{n}\right)-i_{\Phi}\left(w^{n}\right)}, \\
b & =2^{n \tau^{\prime}}
\end{aligned}
$$

the definition of $\mathcal{A}_{\tau}$, with the substitution of (137), yields

$$
\begin{aligned}
\mathbf{1}_{\mathcal{A}_{\tau}}\left(w^{n}, u^{n}, v^{n}\right) & \leq\left(\frac{2^{n \tau^{\prime}}}{2^{i_{\Phi}\left(w^{n}, u^{n} ; v^{n}\right)-i_{\Phi}\left(w^{n}\right)}}\right)^{\beta^{\prime}} \\
& =\left(\frac{2^{n \tau^{\prime}}}{\Phi\left(W^{n}\right) \frac{\Phi\left(V^{n} \mid W^{n}, U^{n}\right)}{\Phi\left(V^{n}\right)}}\right)^{\beta^{\prime}} .
\end{aligned}
$$

We apply (142) to the second term in the bound of Theorem VII.1.

$$
\begin{aligned}
& \delta_{\Phi}(\tau) \\
& =\frac{1}{2} \mathbf{E}_{\Phi_{V^{n}}} \sqrt{\mathbf{E}_{\Phi_{W^{n}, U^{n} \mid V^{n}} \Phi\left(W^{n}\right) \frac{\Phi\left(V^{n} \mid W^{n}, U^{n}\right)}{\Phi\left(V^{n}\right)} \mathbf{A}_{\tau}}} \\
& \leq \frac{1}{2} \mathbf{E}_{\Phi} \sqrt{\mathbf{E}_{\Phi_{\mid V^{n}}} 2^{n \beta^{\prime} \tau^{\prime}}\left(\Phi\left(W^{n}\right) \frac{\Phi\left(V^{n} \mid W^{n}, U^{n}\right)}{\Phi\left(V^{n}\right)}\right)^{1-\beta^{\prime}}} \\
& =\frac{1}{2} \mathbf{E}_{\Phi} \sqrt{\mathbf{E}_{\Phi_{\mid V^{n}}} 2^{n \beta^{\prime} \tau^{\prime}}\left(\prod_{t=1}^{n} \Phi\left(W_{t}\right) \frac{\Phi\left(V_{t} \mid W_{t}, U_{t}\right)}{\Phi\left(V_{t}\right)}\right)^{1-\beta^{\prime}}} \\
& \stackrel{(a)}{=} \frac{1}{2} 2^{\frac{n}{2} \beta^{\prime} \tau^{\prime}} \prod_{t=1}^{n} \mathbf{E}_{\Phi} \sqrt{\mathbf{E}_{\Phi}\left(\Phi\left(W_{t}\right) \frac{\Phi\left(V_{t} \mid W_{t}, U_{t}\right)}{\Phi\left(V_{t}\right)}\right)^{1-\beta^{\prime}}} \\
& \stackrel{(b)}{=} \frac{1}{2} 2^{\frac{n}{2} \beta^{\prime} \tau^{\prime}}\left(\mathbf{E}_{\Phi_{V}} \sqrt{\left.\mathbf{E}_{\Phi_{W, U \mid V}} Z^{1-\beta^{\prime}}\right)^{n}}\right. \\
& =\frac{1}{2} \exp \left(n\left(\frac{1}{2} \beta^{\prime} \tau^{\prime} \log 2+\log \mathbf{E}_{\Phi_{V}} \sqrt{\mathbf{E}_{\Phi_{W, U \mid V} Z^{1-\beta^{\prime}}}}\right)\right),
\end{aligned}
$$

where (a) is from the independence of the distribution at each point in the sequence and (b) uses stationarity. Notice that $\beta^{\prime}=1$ gives the relaxation found in 105 .

Select $\tau^{\prime}$ so that the exponents of (138) and (143) are equal:

$$
\tau^{\prime}=\frac{2 \log \mathbf{E}_{\Phi} Z^{\beta}-2 \log \mathbf{E}_{\Phi_{V}} \sqrt{\mathbf{E}_{\Phi_{W, U \mid V}} Z^{1-\beta^{\prime}}}}{\left(2 \beta+\beta^{\prime}\right) \log 2} .
$$

The common exponent is then

$$
\frac{\beta^{\prime} \log \mathbf{E}_{\Phi} Z^{\beta}+2 \beta \log \mathbf{E}_{\Phi_{V}} \sqrt{\mathbf{E}_{\Phi_{W, U \mid V}} Z^{1-\beta^{\prime}}}}{2 \beta+\beta^{\prime}} .
$$

\section{SUMMARY}

The distributed channel synthesis problem demands unconventional codec constructions, including a stochastic decoder, yet lends itself to a complete information theoretic description of the achievable rate region, found in Theorem II.1. This region reveals that common randomness, independent of the channel input, can replace some of the required communication rate for channel synthesis, reducing the communication rate from Wyner's common information $C(X ; Y)$ to Shannon's mutual information $I(X ; Y)$. Also, §ЩI-E highlights that distributed channel synthesis is as efficient as local channel synthesis in terms of random bits needed by the system.

The main result of Theorem II.1 can be extended to arbitrary channel input sequences, not necessarily i.i.d., and unknown at the time of the codec design. This is shown in [10] using a proof based on the method of types and is also obtained in [54]. The modification needed in our proof is simple but important. Rather than make a statement about the expected total variation vanishing with $n$, a stronger soft covering lemma must instead show that the probability of the total variation exceeding a vanishing threshold goes doubly exponentially to zero, which can be accomplished using the Chernoff bound. This allows the union bound to bridle the exponentially large space of channel input sequences.

Distributed channel synthesis has application to secrecy, game theory, quantum measurements, etc. Additionally, the proof and coding techniques of this work may be of independent interest. In particular, the achievability proof embarks on a construction of a feasible joint distribution over all parts of the system, without first specifying the encoder and decoder behavior. From this, the likelihood encoder is derived. This approach, and the likelihood encoder, can be utilized for problems in information theory in general. Furthermore, this work generalizes and extends the concept of soft covering discussed in $[\mathrm{IV}$ and $\$ \mathrm{VII}$, providing a variety of tools for using codebooks and limit randomness to match an output distribution. In doing so, we derive improved exponents for channel resolvability and, in the appendix, a converse for mean-resolvability of discrete memoryless channels.

\section{APPENDIX}

\section{A. Derivation for Erasure Channel Example of $\$ I I-F$}

For any $P_{X, Y, U} \in \mathcal{D}$, the Markov property constrains that for each value $u$ in the support of $U$ the conditional distribution $P_{X, Y \mid U=u}$ is a product distribution $P_{X \mid U=u} P_{Y \mid U=u}$. These distributions must fall into three categories, shown in Fig. 21, because the events $(X, Y)=(0,1)$ and $(X, Y)=(1,0)$ have zero probability.

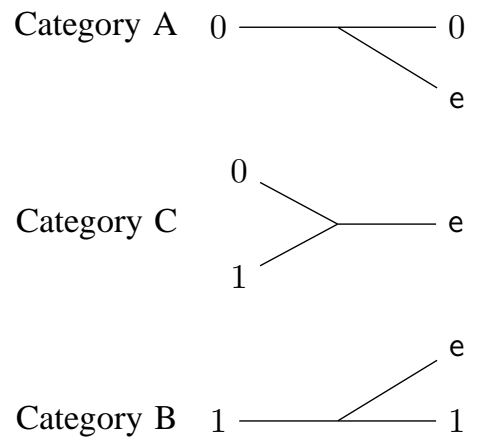

Fig. 21: Categories of Conditional Distributions: Conditional distributions $P_{X, Y \mid U=u}$ for the erasure channel must fit into one of three categories due to the sparsity of the channel.

The three conditional distribution categories are as follows:

- Category A: If $P_{X \mid U=u} P_{Y \mid U=u}$ puts positive probability on $Y=0$, then $X=0$ with probability one. 
- Category B: The reverse occurs if $P_{X \mid U=u} P_{Y \mid U=u}$ puts positive probability on $Y=1$.

- Category C: The only alternative to categories A and B is to put zero probability on $Y \in\{0,1\}$.

We now make two observations and prove them out in the subsequent paragraphs. First, it is sufficient to consider only distributions $P_{X, Y, U} \in \mathcal{D}$ which have at most one value of $u$ for each of the three categories in Fig. 21. Notice that this gives us a bound of $|\mathcal{U}| \leq 3$ for this example, which is less than the nominal bound $|\mathcal{U}| \leq|\mathcal{X}||\mathcal{Y}|+1=7$. Second, the distribution $P_{X, Y, U}$ is symmetric. Therefore, the optimal construction of $P_{X, Y, U}$ for synthesizing the symmetric binary erasure channel for symmetric inputs is a concatenation of two symmetric binary erasure channels, as depicted in Fig. 3 .

Only one of each category: Consider a distribution $P_{X, Y, U}$ where $U$ has two values in its support that are associated with the same category of product distributions (Fig. 21). Define $W=f(U)$ as the label of the distribution category associated with $U$ (i.e. values of $U$ having the same product distribution category map to the same value of $W)$. The data processing inequality says that $I(X ; W) \leq I(X ; U)$ and $I(X, Y ; W) \leq$ $I(X, Y ; U)$. We simply need to verify that $P_{X, Y, W} \in \mathcal{D}-$ in particular, that the Markov chain property $X-W-Y$ holds. This follows because in each category either $X$ or $Y$ is deterministic.

Symmetry: The desired input-output distribution $Q_{X, Y}$ is symmetric. Consider any candidate distribution $P_{X, Y, U} \in \mathcal{D}$, where $\mathcal{U}=\{A, B, C\}$ labels the category of the associated conditional product distribution $P_{X, Y \mid U}$. Define $P_{\tilde{X}, \tilde{Y}, \tilde{U}}$ to be the flipped distribution where $\tilde{X}=1-X, \tilde{Y}=1-Y$, and $\tilde{U}$ is equal to $U$ with $A$ and $B$ exchanged. Clearly $P_{\tilde{X}, \tilde{Y}, \tilde{U}}$ is also in $\mathcal{D}$ and produces the same point in $\mathcal{S}$. It also has the same property that the value of $\tilde{U}$ correctly labels the category of the product distribution. Now define the symmetric distribution $P^{\prime}{ }_{X, Y, U}$ to be the average of $P_{X, Y, U}$ and $P_{\tilde{X}, \tilde{Y}, \tilde{U}}$. Noting that the distribution on $(X, Y)$ is constant within $\mathcal{D}$, the convexity of mutual information with respect to conditional distributions gives $I_{P^{\prime}}(X ; U) \leq I_{P}(X ; U)$ and $I_{P^{\prime}}(X, Y ; U) \leq I_{P}(X, Y ; U)$. Furthermore, $P^{\prime}{ }_{X, Y, U} \in \mathcal{D}$ because mixtures of distributions within a category always result in a product distribution, as discussed above.

\section{B. Proofs for $₫[I I$}

Proof of Lemma [II.1 in $\$$ III-B. The proof that $\mathcal{G} \supset$ Convex $\operatorname{Hull}\left(\mathcal{G}_{0}\right)$ follows naturally from Theorem $\Pi 1.1$ Notice that any point in $\mathcal{G}_{0}$ can be achieved by Player 1 first generating $X^{n}$ and then using the communication to synthesize a channel with output $Y^{n}$. The inequalities in (8) are both satisfied with $\left(R, R_{0}\right)=(C(X ; Y), 0)$. The property of total variation stated in (29) allows us to analyze the payoff as if the actions produced are exactly i.i.d. Then time sharing gives us the convex hull of $\mathcal{G}_{0}$.

For the converse statement, $\mathcal{G} \subset$ Convex $\operatorname{Hull}\left(\mathcal{G}_{0}\right)$, we need to rule out the possibility that some other use of the communication, not resulting in nearly i.i.d. actions, is more beneficial. Let $U$ represent the message used for communication. Notice the following:

$$
\begin{aligned}
n R & \geq H(U) \\
& \geq I\left(U ; X^{n}, Y^{n}\right) \\
& =\sum_{t=1}^{n} I\left(U ; X_{t}, Y_{t} \mid X^{t-1}, Y^{t-1}\right) \\
& =n I\left(U ; X_{T}, Y_{T} \mid X^{T-1}, Y^{T-1}, T\right),
\end{aligned}
$$

and

$$
\begin{aligned}
\Pi & \leq \frac{1}{n} \sum_{t=1}^{n} \Pi_{t} \\
& =\frac{1}{n} \sum_{t=1}^{n} \min _{z(\cdot, \cdot)} \mathbb{E} \pi\left(X_{t}, Y_{t}, z\left(X^{t-1}, Y^{t-1}\right)\right) \\
& =\min _{z(\cdot, \cdot, \cdot)} \frac{1}{n} \sum_{t=1}^{n} \mathbb{E} \pi\left(X_{t}, Y_{t}, z\left(X^{t-1}, Y^{t-1}, t\right)\right) \\
& =\min _{z(\cdot, \cdot, \cdot)} \mathbb{E} \pi\left(X_{T}, Y_{T}, z\left(X^{T-1}, Y^{T-1}, T\right)\right),
\end{aligned}
$$

where $T$ is an independent random variable uniformly distributed on the set $\{1, \ldots, n\}$. For simplicity, let us summarize by making the substitution $W=\left(X^{T-1}, Y^{T-1}, T\right), X=$ $X_{T}$, and $Y=Y_{T}$. Notice that we have the Markov chain $X-(U, W)-Y$ by the constraints of the communication. Then,

$$
\begin{aligned}
& R \geq I(X, Y ; U \mid W), \\
& \Pi \leq \min _{z(\cdot)} \mathbf{E} \pi(X, Y, z(W)) .
\end{aligned}
$$

This is equivalent to the convexification of the points in $\mathcal{G}_{0}$.

Proof of $\$ I I I-C$. Points in $\mathcal{S}_{P C}$ can be achieved the same way as points in $\mathcal{S}$ are achieved for the main result of Theorem II.1 with the additional step of applying a onetime-pad to the communication message.

To prove that this is optimal (converse), we first use the triangle inequality and Lemma $\mathrm{V}$.1 to note that

$$
\begin{gathered}
\left\|P_{X^{n}, Y^{n}, J}-P_{X^{n}, Y^{n}} P_{J}\right\|_{T V} \\
\leq\left\|P_{X^{n}, Y^{n}, J}-P_{J} \prod Q_{X} Q_{Y \mid X}\right\|_{T V} \\
+\left\|P_{X^{n}, Y^{n}} P_{J}-P_{J} \prod Q_{X} Q_{Y \mid X}\right\|_{T V} \\
=\left\|P_{X^{n}, Y^{n}, J}-P_{J} \prod Q_{X} Q_{Y \mid X}\right\|_{T V} \\
+\left\|P_{X^{n}, Y^{n}}-\prod Q_{X} Q_{Y \mid X}\right\|_{T V} \\
\leq 2\left\|P_{X^{n}, Y^{n}, J}-P_{J} \prod Q_{X} Q_{Y \mid X}\right\|_{T V} .
\end{gathered}
$$

By the definition of achievability, the right-hand side can be made arbitrarily small. We next follow the steps of $\$ \mathrm{VI}$ Notice first that we can use Theorem 17.3.3 of [32] to bound the mutual information,

$$
\begin{aligned}
& I\left(X^{n}, Y^{n} ; J\right) \\
& \quad=H\left(X^{n}, Y^{n}\right)+H(J)-H\left(X^{n}, Y^{n}, J\right) \\
& \quad \leq 4 n \epsilon\left(\log |\mathcal{X}|+\log |\mathcal{Y}|+R+\log \frac{1}{\epsilon}\right),
\end{aligned}
$$


where $\epsilon$ is the arbitrarily small total variation tolerance of the synthesis objective.

Now we replace the steps of 86 with

$$
\begin{aligned}
n R_{0} \geq & H_{P}(K) \\
\geq & H_{P}(K \mid J) \\
\geq & I_{P}\left(X^{n}, Y^{n} ; K \mid J\right) \\
\geq & I_{P}\left(X^{n}, Y^{n} ; J, K\right) \\
& \quad-4 n \epsilon\left(\log |\mathcal{X}|+\log |\mathcal{Y}|+R+\log \frac{1}{\epsilon}\right)
\end{aligned}
$$

The proof is completed by following the remaining steps of $\$ \mathrm{VI}$ and altering the definitions of $\mathcal{S}_{\epsilon}$ and $\mathcal{D}_{\epsilon}$ in 80 and appropriately.

Proof of $₫ I I I-D$. To pass statistical tests with limited memory, we use the same construction as in $8 \mathrm{~V}$. Notice that (61) still holds with the rates provided, and we adjust (59) to claim that, uniformly for all $t$,

$$
\lim _{n \rightarrow \infty} \mathbf{E}\left\|\Upsilon_{X_{t-B}^{t}, Y_{t-B}^{t}}-\prod Q_{X} Q_{Y \mid X}\right\|_{T V}=0 .
$$

Furthermore, we call on (56) from Lemma IV.1 to claim that the limits converge exponentially quickly in $n$.

Taking steps analogous to 64,

$$
\begin{aligned}
& \left\|P_{X_{t-B}^{t}, Y_{t-B}^{t}}-\prod Q_{X} Q_{Y \mid X}\right\|_{T V} \\
& \leq\left\|P_{X_{t-B}^{t}, Y_{t-B}^{t}}-\Upsilon_{X_{t-B}^{t}, Y_{t-B}^{t}}\right\|_{T V} \\
& +\left\|\Upsilon_{X_{t-B}^{t}, Y_{t-B}^{t}}-\prod Q_{X} Q_{Y \mid X}\right\|_{T V} \\
& \stackrel{(a)}{\leq}\left\|P_{X^{n}, Y^{n}, J, K}-\Upsilon_{X^{n}, Y^{n}, J, K}\right\|_{T V} \\
& +\left\|\Upsilon_{X_{t-B}^{t}, Y_{t-B}^{t}}-\prod Q_{X} Q_{Y \mid X}\right\|_{T V} \\
& \stackrel{(b)}{=} \quad\left\|P_{X^{n}, K}-\Upsilon_{X^{n}, K}\right\|_{T V} \\
& +\left\|\Upsilon_{X_{t-B}^{t}, Y_{t-B}^{t}}-\prod Q_{X} Q_{Y \mid X}\right\|_{T V} \\
& =\left\|\frac{1}{2^{n R_{0}}}\left(\prod Q_{X}\right)-\Upsilon_{X^{n}, K}\right\|_{T V} \\
& +\left\|\Upsilon_{X_{t-B}^{t}, Y_{t-B}^{t}}-\prod Q_{X} Q_{Y \mid X}\right\|_{T V}
\end{aligned}
$$

where (a) is a consequence of Lemma V.1 and (b) uses Lemma V.2.

The expected value of the right-hand side above goes to zero exponentially fast. Therefore,

$$
\begin{aligned}
\mathbf{E} & \sum_{t=B+1}^{n}\left\|P_{X_{t-B}^{t}, Y_{t-B}^{t}}-\prod Q_{X} Q_{Y \mid X}\right\|_{T V} \\
= & \sum_{t=B+1}^{n} \mathbf{E}\left\|P_{X_{t-B}^{t}, Y_{t-B}^{t}}-\prod Q_{X} Q_{Y \mid X}\right\|_{T V} \\
& \rightarrow 0
\end{aligned}
$$

This confirms the existence of a channel synthesis code that passes limited memory statistical tests for all $t$ simultaneously.
Proof of $\$[I-E$. For limited local randomness, achievability is straightforward. The construction in $\$ \mathrm{~V}$ is valid. We only need to locally synthesize the channel $P_{Y \mid U}$. This can be done with a rate $R_{L}>H(Y \mid U)$ (see Corollary VII.6 of 乌II-B).

For the converse, we modify the proof of the epsilon rate region in Lemma VI.4 with the following argument, where $K_{L}$ represents the local randomness available to the decoder:

$$
\begin{aligned}
n R_{L} & \geq H_{P}\left(K_{L}\right) \\
& \geq I_{P}\left(Y^{n} ; K_{L} \mid J, K\right) \\
& =H_{P}\left(Y^{n} \mid J, K\right) \\
& =\sum_{t=1}^{n} H_{P}\left(Y_{t} \mid J, K, Y^{t-1}\right) \\
& =n H_{P}\left(Y_{T} \mid J, K, Y^{T-1}, T\right) .
\end{aligned}
$$

Notice that $Y^{T-1}$ can be added into (85) alongside $J, K$, and $T$, and also it can be left in the derivation of 86 . Also notice that $X_{T}-\left(J, K, Y^{T-1}, T\right)-Y_{T}$ forms a Markov chain. Therefore, the converse can be complete by replacing $\left(J, K, Y^{T-1}, T\right)$ with $U$ using Lemma VI.1, as in \$VI-C.

\section{Comparison of Soft Covering Lemma to bound in [17]}

The proof of Lemma VII.1 provided in this work, which yields Corollary VII.4 differs from the proof in [17]. Their proof is built around a relationship between the log-likelihood ratio and total variation, encapsulated in Lemma 5 of [17]. Despite some similarity between the proofs, they are fundamentally different and produce different bounds. We can follow the steps of [17] to arrive at an equivalent of Corollary VII.2 and make a straightforward comparison to 106. After making appropriate substitutions in [17],

$$
\begin{aligned}
\mathbf{E} \| P_{V} & -Q_{V} \|_{T V} \\
\leq & \mathbf{P}_{\Phi}\left(i_{\Phi}(U ; V)>\tau\right)+2 \frac{2^{\tau}}{M} \\
& +\mathbf{P}_{\Phi}\left(i_{\Phi}(U ; V)>\log M\right) \\
& +\left(\frac{M}{2^{\tau}}\right)^{2} \mathbf{E} 2^{i_{\Phi}(U ; V)-\log M} \mathbf{1}\left(i_{\Phi}(U ; V) \leq \log M\right) .
\end{aligned}
$$

Consider the four terms in 157). The first two terms are of similar form to (106) and in fact smaller for large $M$. But the second and fourth terms are actually the dominant terms. Using techniques from the proof of Lemma VII.9 it can be shown that for memoryless sources and channels the above bound proves an exponential decay in total variation with respect to the block-length. However, the exponent, given below, is smaller than that of Lemma IV.1

$$
\min _{\alpha \in[1,2]} \frac{\alpha-1}{3}\left(R-\breve{I}_{\Phi, \alpha}(U ; V)\right),
$$

where $\breve{I}_{\Phi, \alpha}(U ; V)$ is defined in (127).

If we follow the method of [17] further, we split the fourth term in 157 into two pieces using an indicator function with a carefully chosen threshold. The result is a simpler bound. 
After substituting $\bar{\tau}=\frac{1}{\eta} \tau+\frac{\eta-1}{\eta} \log M$ for any $\eta \geq 3$ and combining terms, we obtain the following:

$$
\begin{aligned}
\mathbf{E}\left\|P_{V}-Q_{V}\right\|_{T V} \leq & 3\left(\frac{M}{2^{\bar{\tau}}}\right)^{\frac{\eta-1}{\eta}} \mathbf{P}_{\Phi}\left(i_{\Phi_{U, V}}(U ; V)>\bar{\tau}\right) \\
& +3\left(\frac{M}{2^{\bar{\tau}}}\right)^{-\frac{1}{\eta}} \quad \forall \eta \geq 3 .
\end{aligned}
$$

In this form, it is easy to verify that this inequality is dominated by (106) in Corollary VII.2

\section{Mean-resolvability Converse for DMCs}

Many of the soft covering lemmas of $\mathrm{IV}$ and $\$ \mathrm{VII}$ give a tight rate requirement for producing an accurate channel output distribution in the limit of large block-lengths. This input rate requirement is the definition of channel resolvability [17]. Here we demonstrate a simple converse for memoryless channels based on entropy. The following method serves also as a converse for mean-resolvability (measured by entropy of the index to the codebook rather than the logarithm of the cardinality), which settles Remark 4 of [17].

Consider the setting of LemmaIV.1, where $J$ is the stochastic input to a deterministic codebook which produce $u^{n}(J)$ as the input to a memoryless channel specified by $\Phi_{V \mid U}$. Relax the requirement that $J$ is uniformly distributed, but require that $H(J) \leq n R$, where $n$ is the block-length. Consider a desired output distribution $\Phi_{V}$ which is uniquely induced through the channel by an input distribution $\Phi_{U}$ (the proof is easily modified if the input distribution is not unique). We will show that if for all $\epsilon>0$ there exists a block-length $n$, a stochastic input $J$, and a codebook such at that the induced output distribution $P_{V^{n}}$ is $\epsilon$-close to the desired output distribution $Q_{V^{n}}=\prod \Phi_{V}$ as measured by total variation, then $R \geq I_{\Phi}(U ; V)$.

First consider the following entropy manipulation:

$$
\begin{aligned}
H_{P}\left(V^{n}\right) & \leq H_{P}\left(J, U^{n}, V^{n}\right) \\
& =H_{P}\left(J, U^{n}\right)+H_{P}\left(V^{n} \mid U^{n}\right) \\
& =H_{P}(J)+H_{P}\left(V^{n} \mid U^{n}\right) \\
& \leq n R+H_{P}\left(V^{n} \mid U^{n}\right) .
\end{aligned}
$$

By Theorem 17.3.3 of [32], for any $\epsilon<1 / 4$,

$$
\begin{aligned}
H_{P}\left(V^{n}\right) & \geq H_{\Phi}\left(V^{n}\right)-2 \epsilon \log \left(\frac{|\mathcal{V}|^{n}}{\epsilon}\right) \\
& =n H_{\Phi}(V)-2 n \epsilon \log |\mathcal{V}|-2 \epsilon \log \frac{1}{\epsilon} \\
& \geq n\left(H_{\Phi}(V)-2 \epsilon\left(\log |\mathcal{V}|+\log \frac{1}{\epsilon}\right)\right) .
\end{aligned}
$$

Finally, interpret $H_{P}\left(V^{n} \mid U^{n}\right)$ as an expected value of the channel entropy over the input distribution.

$$
\begin{aligned}
H_{P}\left(V^{n} \mid U^{n}\right) & \stackrel{(a)}{=} \sum_{t=1}^{n} H_{P}\left(V_{t} \mid U_{t}\right) \\
& =\mathbf{E}_{P_{U^{n}}} \sum_{t=1}^{n} H_{\Phi}\left(V \mid U_{t}\right) \\
& =\sum_{t=1}^{n} \mathbf{E}_{P_{U_{t}}} H_{\Phi}\left(V \mid U_{t}\right),
\end{aligned}
$$

where (a) is the memoryless property of the channel.

Now we assert that $P_{U_{t}}$ is close to $\Phi_{U}$ in total variation. First notice that $\left\|P_{V_{t}}-\Phi_{V}\right\|_{T V} \leq\left\|P_{V^{n}}-Q_{V^{n}}\right\|_{T V}<\epsilon$ for all $t$ by Lemma V.1. Also, the channel $\Phi_{V \mid U}$ acts as a linear function on the input distributions $P_{U_{t}}$ to produce an output distribution $P_{V_{t}}$. Because the function is continuous on a compact domain, there exists a $\beta(\epsilon)$ which goes to zero as $\epsilon$ goes to zero such that $\left\|P_{U_{t}}-\Phi_{U}\right\|_{T V}<\beta(\epsilon)$.

By the bound in 29, for all $t$,

$$
\mathbf{E}_{P_{U_{t}}} H_{\Phi}\left(V \mid U_{t}\right) \leq \mathbf{E}_{\Phi_{U}} H_{\Phi}(V \mid U)+2 \beta(\epsilon) \log |\mathcal{V}|
$$

Finally, combining inequalities gives

$$
\begin{gathered}
R \geq I_{\Phi}(U ; V)-2 \epsilon\left(\log |\mathcal{V}|+\log \frac{1}{\epsilon}\right) \\
-2 \beta(\epsilon) \log |\mathcal{V}| .
\end{gathered}
$$

Since this statement is true for all $\epsilon \in(0,1 / 4)$, we conclude that $R \geq I_{\Phi}(U ; V)$.

\section{REFERENCES}

[1] P. Cuff, "Communication requirements for generating correlated random variables," in IEEE Int'l. Symp. on Inf. Theory (ISIT), July 2008.

[2] P. Cuff, H. Permuter, and T. Cover, "Coordination capacity," IEEE Trans. Inf. Theory, vol. 56, no. 9, pp. 4181-4206, Sept. 2010.

[3] V. Anantharam and V. Borkar, "Common randomness and distributed control: A counterexample," Systems \& Control Letters, vol. 56, no. 7-8, pp. 568-572, 2007. [Online]. Available: http://www.sciencedirect.com/science/article/pii/S0167691107000540

[4] A. Gilpin and T. Sandholm, "Solving two-person zero-sum repeated games of incomplete information," in 7th international joint conference on autonomous agents and multiagent systems (AAMAS), 2008.

[5] P. Gács and J. Körner, "Common information is far less than mutual information," Problems of Control and Inf. Theory, vol. 2, pp. 149-162, 1973.

[6] A. Wyner, "The common information of two dependent random variables," IEEE Trans. Inf. Theory, vol. 21, no. 2, pp. 163-179, March 1975.

[7] C. Bennett, P. Shor, J. Smolin, and A. Thapliyal, "Entanglement-assisted capacity of a quantum channel and the reverse shannon theorem," IEEE Trans. Inf. Theory, vol. 48, no. 10, pp. 2637-2655, Oct. 2002.

[8] E. Soljanin, "Compressing quantum mixed-state sources by sending classical information," IEEE Trans. Inf. Theory, vol. 48, no. 8, pp. 2263 2275, 2002.

[9] C. Bennett, P. Shor, J. Smolin, and A. Thapliyal, "Entanglementassisted classical capacity of noisy quantum channels," Phys. Rev. Lett., vol. 83, pp. 3081-3084, Oct. 1999. [Online]. Available: http://link.aps.org/doi/10.1103/PhysRevLett.83.3081

[10] C. Bennett, I. Devetak, A. Harrow, P. Shor, and W. A., "Quantum reverse shannon theorem," April 2012, submitted to IEEE Trans. Inf. Theory, arXiv:0912.5537.

[11] M. Berta, M. Christandl, and R. Renner, "The quantum reverse shannon theorem based on one-shot information theory," Communications in Mathematical Physics, vol. 306, no. 3, pp. 579-615, 2011. [Online]. Available: http://dx.doi.org/10.1007/s00220-011-1309-7

[12] A. Winter, "Compression of sources of probability distributions and density operators," Aug. 2002, arXiv:quant-ph/0208131.

[13] —, "Extrinsic and intrinsic data in quantum measurements: Asymptotic convex decomposition of positive operator valued measures," Communications in Mathematical Physics, vol. 244, pp. 157-185, 2004, 10.1007/s00220-003-0989-z. [Online]. Available: http://dx.doi.org/10.1007/s00220-003-0989-z

[14] M. Wilde, P. Hayden, F. Buscemi, and M.-H. Hsieh, "The informationtheoretic costs of simulating quantum measurements," Journal of Physics A: Mathematical and Theoretical, vol. 45, no. 45, p. 453001, 2012. [Online]. Available: http://stacks.iop.org/1751-8121/45/i=45/a=453001

[15] P. Harsha, R. Jain, D. McAllester, and J. Radhakrishnan, "The communication complexity of correlation," in Twenty-Second Annual IEEE Conference on Computational Complexity (CCC), June 2007. 
[16] T. Cubitt, D. Leung, W. Matthews, and A. Winter, "Zero-error channel capacity and simulation assisted by non-local correlations," IEEE Trans. Inf. Theory, vol. 57, no. 8, pp. 5509-5523, Aug. 2011.

[17] T. Han and S. Verdú, "Approximation theory of output statistics," IEEE Trans. Inf. Theory, vol. 39, no. 3, pp. 752-772, May 1993.

[18] A. Gohari and V. Anantharam, "Generating dependent random variables over networks," in IEEE Information Theory Workshop (ITW), Oct. 2011.

[19] M. Yassaee, M. Aref, and A. Gohari, "Achievability proof via output statistics of random binning," in IEEE Int'l. Symp. on Inf. Theory (ISIT), July 2012.

[20] M. Yassaee, A. Gohari, and M. Aref, "Channel simulation via interactive communications," in IEEE Int'l. Symp. on Inf. Theory (ISIT), July 2012.

[21] F. Haddadpour, M. Yassaee, A. Gohari, and M. Aref, "Coordination via a relay," in IEEE Int'l. Symp. on Inf. Theory (ISIT), July 2012.

[22] S. Satpathy and P. Cuff, "Secure cascade channel synthesis," in IEEE Int'l. Symp. on Inf. Theory (ISIT), July 2013.

[23] P. Cuff, "Communication in networks for coordinating behavior," Ph.D. dissertation, Stanford University, Aug. 2009.

[24] C. Bennett, I. Devetak, A. Harrow, P. Shor, and A. Winter, "Quantum reverse shannon theorem," 2007, presentation: http://www.research.ibm.com/people/b/bennetc/QRSTonlineVersion.pdf.

[25] H. Witsenhausen, "Values and bounds for the common information of two discrete random variables," SIAM Journal on Applied Mathematics, vol. 31, no. 2, pp. 313-333, 1976. [Online]. Available: http://epubs.siam.org/doi/abs/10.1137/0131026

[26] A. Winter, "Secret, public and quantum correlation cost of triples of random variables," in IEEE Int'l. Symp. on Inf. Theory (ISIT), Sept. 2005.

[27] P. Cuff, "A framework for partial secrecy," in IEEE Global Telecommunications Conference (GLOBECOM), Dec. 2010.

[28] - "Using a secret key to foil an eavesdropper," in 48th Annual Allerton Conference on Communication, Control, and Computing (Allerton), Oct. 2010.

[29] C. Schieler and P. Cuff, "Secrecy is cheap if the adversary must reconstruct," in IEEE Int'l. Symp. on Inf. Theory (ISIT), July 2012.

[30] Y. Steinberg and S. Verdú, "Simulation of random processes and ratedistortion theory," IEEE Trans. Inf. Theory, vol. 42, no. 1, pp. 63-86, Jan. 1996

[31] M. Bloch and J. N. Laneman, "Secrecy from resolvability," 2011, submitted to IEEE Trans. Inf. Theory, arXiv:1105.5419.

[32] T. Cover and J. Thomas, Elements of Information Theory (Wiley Series in Telecommunications and Signal Processing). Wiley-Interscience, 2006.

[33] P. Cuff, "State information in bayesian games," Nov. 2009, presented at Allerton, arXiv:0911.0874.

[34] M. Bloch and J. Kliewer, "On secure communication with constrained randomization," in IEEE Int'l. Symp. on Inf. Theory (ISIT), July 2012.

[35] Y. Steinberg and S. Verdú, "Channel simulation and coding with side information," IEEE Trans. Inf. Theory, vol. 40, no. 3, pp. 634-646, May 1994.

[36] R. Gray and A. Wyner, "Source coding for a simple network," Bell Systems Technical Journal, vol. 53, no. 9, pp. 1681-1721, Nov. 1974.
[37] U. Maurer, "Secret key agreement by public discussion from common information," IEEE Trans. Inf. Theory, vol. 39, no. 3, pp. 733-742, May 1993.

[38] R. Ahlswede and I. Csiszár, "Common randomness in information theory and cryptography. i. secret sharing," IEEE Trans. Inf. Theory, vol. 39, no. 4, pp. 1121-1132, July 1993

[39] - "Common randomness in information theory and cryptography. ii. cr capacity," IEEE Trans. Inf. Theory, vol. 44, no. 1, pp. 225-240, Jan. 1998.

[40] I. Csiszár and P. Narayan, "Common randomness and secret key generation with a helper," IEEE Trans. Inf. Theory, vol. 46, no. 2, pp. 344-366, March 2000.

[41] U. Maurer and S. Wolf, "Information-theoretic key agreement: From weak to strong secrecy for free," in Advances in Cryptology EUROCRYPT 2000, ser. Lecture Notes in Computer Science, B. Preneel, Ed. Springer Berlin / Heidelberg, 2000, vol. 1807, pp. 351-368.

[42] M. Hayashi, "General nonasymptotic and asymptotic formulas in channel resolvability and identification capacity and their application to the wiretap channel," IEEE Trans. Inf. Theory, vol. 52, no. 4, pp. 1562$1575,2006$.

[43] R. Ahlswede and A. Winter, "Strong converse for identification via quantum channels," IEEE Trans. Inf. Theory, vol. 48, no. 3, pp. 569-579, 2002.

[44] M. Wilde, "From classical to quantum shannon theory," 2011, arXiv:1106.1445.

[45] C. Schieler and P. Cuff, "A connection between good rate-distortion codes and backward dmcs," in IEEE Information Theory Workshop (ITW), Sept. 2013.

[46] C. Carathodory, "Über den variabilitätsbereich der fourier'schen konstanten von positiven harmonischen funktionen," Rendiconti del Circolo Matematico di Palermo (1884 - 1940), vol. 32, pp. 193-217, 1911, 10.1007/BF03014795. [Online]. Available: http://dx.doi.org/10.1007/BF03014795

[47] E. Steinitz, "Bedingt konvergente reihen und konvexe systeme," J. Reine Angew. Math., vol. 143, pp. 128-175, 1913.

[48] H. Eggleston, Convexity. Cambridge University Press, 1963.

[49] M. Salehi, "Cardinality bounds on auxiliary variables in multiple-user theory via the method of ahlswede and körner," Technical Report, Stanford University, no. 33, Aug. 1978.

[50] I. Csiszár and J. Körner, Information Theory: Coding Theorems for Discrete Memoryless Systems, 2nd ed. Cambridge University Press, 2011.

[51] A. El Gamal and Y.-H. Kim, Network Information Theory. Cambridge University Press, 2011.

[52] T. Han, Information-spectrum methods in information theory, ser. Applications of Mathematics. Springer, 2003, vol. 50.

[53] I. Csiszár, "Generalized cutoff rates and Rényi's information measures," IEEE Trans. Inf. Theory, vol. 41, no. 1, pp. 26-34, 1995.

[54] M. Berta, J. Renes, and M. Wilde, "Identifying the information gain of a quantum measurement," January 2013, arXiv:1301.1594. 